This is an Accepted Manuscript of an article published by Taylor \& Francis in "Experimental mathematics" on $05^{\text {th }}$ April 2016, available online: http://www.tandfonline.com/doi/full/10.1080/10586458.2015.1076361 


\title{
Exponentially small asymptotic formulas for the length spectrum in some billiard tables
}

\author{
P. Martín , R. Ramírez-Ros ${ }^{\mathrm{a}, *}$, A. Tamarit-Sariol ${ }^{\mathrm{a}}$ \\ ${ }^{a}$ Departament de Matemàtica Aplicada I, Universitat Politècnica de Catalunya, Diagonal 647, 08028 Barcelona, Spain \\ ${ }^{b}$ Departament de Matemàtica Aplicada IV, Universitat Politècnica de Catalunya, Campus Diagonal Nord, Edifici C3. C. Jordi Girona, 1-3, 08034 Barcelona, Spain
}

\begin{abstract}
Let $q \geq 3$ be a period. There are at least two $(1, q)$-periodic trajectories inside any smooth strictly convex billiard table. We quantify the chaotic dynamics of axisymmetric billiard tables close to their boundaries by studying the asymptotic behavior of the differences of the lengths of their axisymmetric $(1, q)$-periodic trajectories as $q \rightarrow+\infty$. Based on numerical experiments, we conjecture that, if the billiard table is a generic axisymmetric analytic strictly convex curve, then these differences behave asymptotically like an exponentially small factor $q^{-3} \mathrm{e}^{-r q}$ times either a constant or an oscillating function, and the exponent $r$ is half of the radius of convergence of the Borel transform of the well-known asymptotic series for the lengths of the $(1, q)$-periodic trajectories. Our experiments are focused on some perturbed ellipses and circles, so we can compare the numerical results with some analytical predictions obtained by Melnikov methods. We also detect some non-generic behaviors due to the presence of extra symmetries. Our computations require a multiple-precision arithmetic and have been programmed in PARI/GP.
\end{abstract}

Keywords: Billiards; Length spectrum; Exponentially small phenomena; Numeric experiments; Melnikov method

\section{Introduction}

Billiards as a dynamical system go back to Birkhoff [1]. Let $Q$ be a closed smooth strictly convex curve in the Euclidean plane. The Birkhoff billiard models the motion of a particle inside the region enclosed by $Q$. The particle moves with unit velocity and without friction following a straight line; it reflects elastically when it hits $Q$. Therefore, billiard trajectories consist of polygonal lines inscribed in $Q$ whose consecutive sides obey to the rule "the angle of reflection is equal to the angle of incidence." Such trajectories are sometimes called broken geodesics. See [2-4] for a general description.

A $(p, q)$-periodic billiard trajectory forms a closed polygon of $q$ sides that makes $p$ turns before closing. Birkhoff [1] proved that there are at least two different Birkhoff $(p, q)$-periodic billiard trajectories inside $Q$ for any relatively prime integers $p$ and $q$ such that $1 \leq p<q$.

The length spectrum of $Q$ is the subset of $\mathbb{R}_{+}$defined as

$$
\mathcal{L S}(Q)=l \mathbb{N} \cup \bigcup_{(p, q)} \Lambda^{(p, q)} \mathbb{N}
$$

where $l=\operatorname{Length}(Q)$ and $\Lambda^{(p, q)} \subset \mathbb{R}_{+}$is the set of the lengths of all $(p, q)$-periodic billiard trajectories inside $Q$. The maximal

\footnotetext{
The authors were supported in part by CUR-DIUE Grant 2014SGR504 (Catalonia) and MINECO-FEDER Grant MTM2012-31714 (Spain). We acknowledge the use of the UPC Applied Math cluster system for research computing.

${ }^{*}$ Corresponding author

Email addresses: martin@ma4.upc.edu (P. Martín), rafael.ramirez@upc. edu (R. Ramírez-Ros), anna.tamarit@upc. edu (A. Tamarit-Sariol)
}

difference among lengths of $(p, q)$-periodic trajectories is the non-negative quantity

$$
\Delta^{(p, q)}=\sup \Lambda^{(p, q)}-\inf \Lambda^{(p, q)} .
$$

Many geometric and dynamical properties are encoded in the length spectrum $\mathcal{L S}(Q)$ and the differences $\Delta^{(p, q)}$.

An old geometric question is: Does the set $\mathcal{L} \mathcal{S}(Q)$ allow one to reconstruct the convex curve $Q$ ? The length spectrum and the Laplacian spectrum with Dirichlet boundary conditions are closely related [5]. Therefore, the question above can be colorfully restated as [6]: Can one hear the shape of a drum? We refer to the book [7] for some results on this question.

The difference $\Delta^{(p, q)}$ is important from a dynamical point of view, because it is an upper bound of Mather's $\Delta W_{p / q}$. In its turn, $\Delta W_{p / q}$ is equal to the flux through the $(p, q)$-resonance of the corresponding billiard map [8-11]. Thus, the variation of $\Delta^{(p, q)}$ in terms of the rotation number $p / q \in(0,1)$ gives information about the size of the different chaotic zones of the billiard map. See Section 2 for a more complete description of these ideas.

Here, our main goal is to gain some insight into the billiard dynamics close to the boundary of the billiard table. We focus on the $(1, q)$-periodic billiards trajectories; that is, we set $p=1$. We want to determine the asymptotic behavior of

$$
\Delta^{(1, q)}=\sup \Lambda^{(1, q)}-\inf \Lambda^{(1, q)}
$$

as $q \rightarrow+\infty$.

Let $L^{(1, q)} \in \Lambda^{(1, q)}$ be the length of a $(1, q)$-periodic billiard trajectory inside $Q$. It does not matter which one. Marvizi and Melrose [12] proved that if $Q$ is smooth and strictly convex, then there exist some asymptotic coefficients $l_{j}=l_{j}(Q)$ such 
that

$$
L^{(1, q)} \asymp \sum_{j \geq 0} l_{j} q^{-2 j}, \quad q \rightarrow \infty .
$$

For instance, $l_{0}=l=\operatorname{Length}(Q)$ and $l_{1}=-\frac{1}{24}\left(\int_{Q} \kappa^{2 / 3} \mathrm{~d} s\right)^{3}$, where $\kappa$ and $\mathrm{d} s$ are the curvature and the length element of $Q$, respectively. The symbol $\asymp$ means that the series in the right hand side is asymptotic to $L^{(1, q)}$. That is, for any order $J \geq 0$, there exists $K_{J}>0$ such that $\left|L^{(1, q)}-\sum_{j=0}^{J} l_{j} q^{-2 j}\right| \leq K_{J} q^{-2 J-2}$ for all period $q \geq 3$. The asymptotic coefficients $l_{j}$ can be explicitly written as integrals over $Q$ of suitable algebraic expressions of $\kappa$ and its derivatives. The first five coefficients can be found in [13]. The asymptotic series (1) does not depend on the choice $L^{(1, q)} \in \Lambda^{(1, q)}$, so

$$
\lim _{q \rightarrow+\infty} q^{k} \Delta^{(1, q)}=0, \quad \forall k>0 .
$$

That is, the differences $\Delta^{(1, q)}$ are beyond all order in $q$. In fact, they satisfy the following exponentially small upper bound in the analytic case [14]. If $Q$ is a closed analytic strictly convex curve, then there exist constants $K, q_{0}, \alpha>0$ such that

$$
\Delta^{(1, q)} \leq K \mathrm{e}^{-2 \pi \alpha q}, \quad \forall q \geq q_{0} .
$$

The exponent $\alpha$ is related to the width of the complex strip where a certain 1-periodic angular coordinate is analytic. If a billiard map (or any analytic exact twist map) has a rotational invariant circle of Diophantine rotation number $\omega$, there exist other exponentially small upper bounds for $\Delta^{(p, q)}$ (or for the residues of $(p, q)$-periodic orbits) when $p / q \rightarrow \omega$. See [15-17].

Similar singular behaviors have been observed in problems about the splitting of separatrices of analytic maps [18-29]. All these splittings are not exponentially small in a discrete big parameter $q \in \mathbb{N}$, but in a continuous small parameter $h>0$. Namely, $h$ is the characteristic exponent of the hyperbolic fixed point whose separatrices split. Thus, we may think that $h=1 / q$ for comparison purposes. The splitting size in many analytic maps satisfies the exponentially small asymptotic formula

$$
\text { “splitting size" } \asymp A(1 / h) h^{-m} \mathrm{e}^{-r / h}, \quad h \rightarrow 0^{+},
$$

for some exponent $r>0$, some power $m \in \mathbb{R}$, and some function $A(1 / h)$ that is either constant or oscillating. The exponent $r$ and the function $A(1 / h)$ are determined by looking at the complex singularities closest to the real axis of the homoclinic solution of a limit Hamiltonian flow related to the map. Such methodology has been rigorously established for the standard map [22], the Hénon map [24], and some perturbed McMillan maps $[20,27,28]$. It has also been numerically checked in certain billiard maps [25] and several polynomial maps [26], but there are other maps where it fails [29]. Let us briefly recall some claims about polynomial standard maps contained in $[19,26]$. First, $r=2 \pi \delta$, where $\delta$ is the distance of these singularities to the real axis. Besides,

$$
A(1 / h)=\mu a / 2+a \sum_{j=1}^{J} \cos \left(2 \pi \beta_{j} / h+\varphi_{j}\right),
$$

for some $\mu \in\{0,1\}$, some amplitude $a \neq 0$, and some phases $\varphi_{j} \in \mathbb{R}$, when these singularities are

$$
\pm \delta \mathrm{i} \text { (if and only if } \mu=1), \pm \beta_{1} \pm \delta \mathrm{i}, \ldots, \pm \beta_{J} \pm \delta \mathrm{i} \text {. }
$$

For instance, the limit Hamiltonian flow for the standard map is a pendulum, so $\pm \pi \mathrm{i} / 2$ are the closest singularities to the real axis and the "splitting size" is the so-called Lazutkin constant $\omega_{0} \simeq 1118.827706$ times $h^{-2} \mathrm{e}^{-\pi^{2} / h}$, see [22].

It is also known that, usually, $r=\rho / 2$, where $\rho$ is the radius of convergence of the Borel transform of the divergent asymptotic series that approaches the separatrices [21, 24-26].

By looking at our billiard problem from the perspective of those results (and others not mentioned here for the sake of brevity), it is natural to make the following conjecture. This conjecture is strongly supported by our numerical experiments.

Conjecture 1. If $Q$ is a closed analytic strictly convex curve, but it is neither a circle nor an ellipse, the asymptotic series (1) diverges for all period $q \in \mathbb{N}$, but it is Gevrey-1, so its Borel transform

$$
\sum_{j \geq 0} \hat{l}_{j} z^{2 j-1}, \quad \hat{l}_{j}=\frac{l_{j}}{(2 j-1) !},
$$

has a radius of convergence $\rho \in(0,+\infty)$. Set $r=\rho / 2$.

If $Q$ is a generic axisymmetric algebraic curve, then

$$
\Delta^{(1, q)} \asymp|A(q)| q^{-3} \mathrm{e}^{-r q}, \quad q \rightarrow+\infty,
$$

for some function $A(q)$ that is either constant: $A(q)=a / 2 \neq 0$, or oscillating:

$$
A(q)=\mu a / 2+a \sum_{j=1}^{J} \cos \left(2 \pi \beta_{j} q\right)
$$

with $\mu \in\{0,1\}, a \neq 0, J \geq 1$, and $0<\beta_{1}<\cdots<\beta_{J}$. The cases $A(q)=a / 2$ and $A(q)=a \cos (2 \pi \beta)$ take place in open sets of the space of axisymmetric algebraic curves. All the other cases are phenomena of co-dimension at least one.

If $Q$ is a generic bi-axisymmetric algebraic curve, $\Delta^{(1, q)}$ has the previous asymptotic behavior when $q$ is even and $q \rightarrow+\infty$, but $\Delta^{(1, q)}=\mathrm{O}\left(q^{-2} \mathrm{e}^{-2 r q}\right)$ when $q$ is odd and $q \rightarrow+\infty$.

We stress that the oscillating function (3) has some phases, but there are no phases in the oscillating function (6). This phenomenon is not new. The asymptotic formulas for the exponentially small splittings of generalized standard maps with trigonometric polynomials do not have phases either [26].

A curve is axisymmetric when it is symmetric with respect to a line, and bi-axisymmetric when it is symmetric with respect to two perpendicular lines. A planar curve is algebraic when its points are the zeros of some polynomial in two variables. We require strict convexity, since it is already an essential hypothesis in the smooth setup. We only consider algebraic curves by comparison with the above results about polynomial standard maps. Our algebraic curves have no singular points, because we ask them to be closed and analytic. 
If $Q$ is a circle of radius $r_{0}$, all its $(p, q)$-periodic billiard trajectories have length $2 r_{0} q \sin (\pi p / q)$, so $\Delta^{(p, q)}=0$ for all $p / q \in(0,1)$, and the asymptotic series (1) becomes

$$
L^{(1, q)}=2 r_{0} q \sin (\pi / q)=2 r_{0} \sum_{j \geq 0} \frac{(-1)^{j} \pi^{2 j+1}}{(2 j+1) !} q^{-2 j},
$$

which converges for all $q$. In particular, $\rho=+\infty$. Ellipses have analogous properties. This has to do with the fact that elliptic and circular billiards are integrable. A conjecture attributed to Birkhoff claims that the only integrable smooth convex billiard tables are ellipses and circles [30]. Following the discussion on the Mather's $\beta$-function contained in [13], this old conjecture is reformulated as: The series in (1) converges for some period $q \in \mathbb{N}$ if and only if $Q$ is an ellipse or a circle.

In this paper, we present several numerical experiments and some analytical results that support Conjecture 1. For the sake of simplicity, all numerical experiments are carried out using the model tables

$$
Q=\left\{(x, y) \in \mathbb{R}^{2}: x^{2}+y^{2} / b^{2}+\epsilon y^{n}=1\right\} .
$$

Here, $b \in(0,1]$ is the semi-minor axis, $\epsilon \in \mathbb{R}$ is the perturbative parameter, and $n \in \mathbb{N}$, with $3 \leq n \leq 8$, is the degree of the perturbation. We will refer to $Q$ as a perturbed ellipse when $0<b<1$ and as a perturbed circle when $b=1$. Next, we explain the four main reasons for this choice of billiard tables.

As a first reason, we know that all the billiard tables (7) are nonintegrable for $n \geq 3$ and $0<\epsilon \ll 1$, and so the dynamics inside them should be far from trivial. The question of which perturbed ellipses give rise to integrable billiards is addressed in [31]. Theorem 4.1 of that paper imply that the tables (7) are nonintegrable if $0<b<1, n \geq 4$ is even, and $\epsilon$ is small enough. This result can be extended, after some technicalities, to odd degrees. Furthermore, all integrable deformations of ellipses of small eccentricities - this includes, of course, circles - are ellipses [32], so the tables (7) are nonintegrable if $b=1, n \geq 3$, and $\epsilon$ is small enough.

The second reason is that we want to use some Melnikov methods that are well suited for the study of billiards inside perturbed ellipses and perturbed circles $[33,34]$. We recall that $\Delta^{(1, q)}=0$ for any $q \geq 3$ in elliptic billiards. Besides, from classical Melnikov theory, we deduce that

$$
\Delta^{(1, q)}=\Delta^{(1, q)}(\epsilon)=\epsilon \Delta_{1}^{(1, q)}+\mathrm{o}(\epsilon)
$$

for some coefficient $\Delta_{1}^{(1, q)} \in \mathbb{R}$ that can be computed explicitly. To be precise, it turns out that if $0<b<1$ then

$$
\Delta_{1}^{(1, q)} \asymp M_{n} q^{m_{n}} \mathrm{e}^{-c q}, \quad q \rightarrow+\infty,
$$

for some Melnikov exponent $c>0$ not depending on $n$, some Melnikov power $m_{n} \in \mathbb{Z}$, and some Melnikov constant $M_{n} \neq 0$. These three Melnikov quantities can be explicitly computed, but we have carried out the computations only for the cubic $(n=3)$ and quartic $(n=4)$ perturbations for the sake of brevity. Besides, $\lim _{b \rightarrow 1^{-}} c=+\infty$. The Melnikov method provides no information when $n$ is odd and $q$ even; $\Delta_{1}^{(1, q)}=0$ in such case. See Proposition 2 for details.
Which is the relation between the asymptotic formula (5) and the first order Melnikov computation (8)? The answer is that $r \neq c$ and $m_{n} \neq-3$, so the Melnikov method does not accurately predict the singular behavior of $\Delta^{(1, q)}$. Nevertheless, $\lim _{\epsilon \rightarrow 0} r=c$, so some information can be retrieved from the Melnikov method, at least for perturbed ellipses.

The case of perturbed circles is harder. See Section 6.

Symmetries are another reason for the choice of tables (7). On the one hand, symmetries greatly simplify the computation of periodic trajectories. To be precise, we just compute the signed difference $D_{q}$ between two particular axisymmetric $(1, q)$-periodic trajectories, instead of $\Delta^{(1, q)}$ or $\Delta W_{1 / q}$. Clearly, $\left|D_{q}\right| \leq \Delta^{(1, q)}$. Often, $\left|D_{q}\right|=\Delta^{(1, q)}=\Delta W_{1 / q}$. See Proposition 2 . On the other hand, bi-axisymmetric curves are a very particular class of axisymmetric curves, so our model tables may display other asymptotic behaviors when $n$ is even. We will check that this expectation is fulfilled. Concretely,

$$
\Delta^{(1, q)} \asymp|B(q)| q^{-2} \mathrm{e}^{-2 r q}, \quad q \rightarrow+\infty,
$$

for some constant or oscillating function $B(q)$ when $n$ is even and $q$ is odd. This asymptotic behavior has several differences with respect to the generic one conjectured in (5). Both the exponent in $\mathrm{e}^{-r q}$ and (if any) the frequencies $0<\beta_{1}<\cdots<\beta_{J}$ are doubled, the power in $q^{-3}$ is increased by one, etcetera. We think that this new asymptotic behavior is generic among biaxisymmetric algebraic curves when the period $q$ is odd.

The last reason for the choice of such simple billiard tables is to reduce the computational effort as much as possible. In particular, we limit the degree of the perturbation to the range $3 \leq n \leq 8$ for this reason. Recall that each set $\Lambda^{(1, q)}$ is contained in an exponentially small (in $q$ ) interval, so the computation of $\Delta^{(1, q)}$ (or $D_{q}$ ) gives rise to very strong cancellations. This forces us to use a multiple-precision arithmetic to compute them. We have performed some computations with more than twelve thousand digits, based on the open source PARI/GP system [35]. Similar computations in the setting of splitting of separatrices of analytic maps can be found in $[21,25,26]$.

Finally, we recall that the exponent $r$ is found by looking at the complex singularities of the homoclinic solution of a limit Hamiltonian flow in many cases of splitting of separatrices. Does such kind of limit problem exist in our billiard setting? Unfortunately, we do not have a completely satisfactory answer yet, but we propose a candidate in Section 3. It is empirically derived by using the Taylor expansions of the billiard dynamics close to the boundary given by Lazutkin in [36]. Let $\kappa(s)$ be the curvature of $Q$ in some arc-length parameter $s \in \mathbb{R} / l \mathbb{Z}$. Let $\xi \in \mathbb{R} / \mathbb{Z}$ be a new angular variable defined by

$$
C \frac{\mathrm{d} \xi}{\mathrm{d} s}=\kappa^{2 / 3}(s), \quad C=\int_{Q} \kappa^{2 / 3} \mathrm{~d} s .
$$

The constant $C$ is sometimes called the Lazutkin perimeter. It is chosen so that $\xi$ has period one. Let $\delta$ be the distance of the set of singularities and zeros of the curvature $\kappa(\xi)$ to the real axis. We thought that $r=2 \pi \delta$, but our experiments disprove it. We have only obtained that $r \leq 2 \pi \delta$, the equality being an infrequent situation. But there are some good news about 
our candidate. First, the Melnikov exponent is $c=2 \pi \delta$, when $Q=\left\{(x, y) \in \mathbb{R}^{2}: x^{2}+y^{2} / b^{2}=1\right\}$, with $0<b<1$. See Proposition 4. Second, we have also seen that, if $b=1$ and $n \geq 3$ is fixed, then there exist some constants $\chi_{n}, \eta_{n} \in \mathbb{R}$, $\chi_{n} \leq \eta_{n}$, such that

$$
r=\frac{|\log \epsilon|}{n}+\chi_{n}+\mathrm{o}(1), \quad 2 \pi \delta=\frac{|\log \epsilon|}{n}+\eta_{n}+\mathrm{o}(1),
$$

as $\epsilon \rightarrow 0^{+}$. The second formula is proved in Proposition 7, the first one is numerically checked in Section 6. Therefore, our candidate exactly captures the logarithmic growth of the exponent $r$ for perturbed circles. Third, our experiments suggest that $r=2 \pi \delta$ when $b=1, n \in\{5,7\}$, and $\epsilon \in(0,1 / 10]$.

The paper has the following structure. Section 2 contains the dynamical interpretation of Mather's $\Delta W_{p / q}$. We discuss our candidate for limit problem in Section 3 . The axisymmetric tables and their axisymmetric periodic billiard trajectories are presented in more detail in Section 4. The main results about perturbed ellipses and perturbed circles are described in Sections 5 and 6, respectively. All proofs have been relegated to the appendices.

\section{Twist maps, actions, Mather's $\Delta W$, and billiards}

We recall some results about exact twist maps and billiards. We refer to the books [2-4] and the surveys [10, 11] for a more detailed exposition.

Let $\mathbb{T}=\mathbb{R} / \mathbb{Z}$ and $I=\left(y_{-}, y_{+}\right) \subset \mathbb{R}$ for some $-\infty \leq y_{-}<$ $y_{+} \leq+\infty$. Let $\omega=\mathrm{d} x \wedge \mathrm{d} y$ be the canonical area form on $\mathbb{T} \times I$. Note that $\omega=-\mathrm{d} \lambda$, where $\lambda=y \mathrm{~d} x$. A smooth diffeomorphism $f: \mathbb{T} \times I \rightarrow \mathbb{T} \times I$ is an exact twist map when it preserves $\omega$, has zero flux, and satisfies the twist condition $\frac{\partial x_{1}}{\partial y}>0$, where $F: \mathbb{R} \times I \rightarrow \mathbb{R} \times I, F(x, y)=\left(x_{1}, y_{1}\right)$, is a (fixed) lift of $f$.

We also assume that $f$ can be extended as rigid rotations of angles $\varrho_{ \pm}$to the boundaries $C_{ \pm}=\mathbb{T} \times\left\{y_{ \pm}\right\}$. We know that $\varrho_{-}<\varrho_{+}$from the twist condition. Let $E=\left\{\left(x, x_{1}\right) \in \mathbb{R}^{2}: \varrho_{-}<\right.$ $\left.x_{1}-x<\varrho_{+}\right\}$. Then there exists a function $h: E \rightarrow \mathbb{R}$ such that $h\left(x+1, x_{1}+1\right)=h\left(x, x_{1}\right)$ and

$$
y_{1} \mathrm{~d} x_{1}-y \mathrm{~d} x=\mathrm{d} h\left(x, x_{1}\right) .
$$

This function is called Lagrangian or generating function. It is determined modulo an additive constant. Twist maps satisfy the following classical Lagrangian formulation. Their orbits are in one-to-one correspondence with the (formal) stationary configurations of the action functional

$$
\mathbb{R}^{\mathbb{Z}} \ni \mathbf{x}=\left(x_{j}\right)_{j \in \mathbb{Z}} \mapsto W[\mathbf{x}]=\sum_{j \in \mathbb{Z}} h\left(x_{j}, x_{j+1}\right) .
$$

Note that, although the series for $W[\mathbf{x}]$ may be divergent, $\frac{\partial W}{\partial x_{j}}$ only involves two terms of the series, and so $\nabla W$ is well defined.

Let $p$ and $q$ be two relatively prime integers such that $p / q \in$ $\left(\varrho_{-}, \varrho_{+}\right)$and $q \geq 1$. A point $(x, y) \in \mathbb{R} \times I$ is $(p, q)$-periodic when $F^{q}(x, y)=(x+p, y)$. The corresponding point $(x, y) \in \mathbb{T} \times I$ is a $q$-periodic point of $f$ that is translated $p$ units in the base by the lift. A $(p, q)$-periodic orbit is Birkhoff when it is ordered around

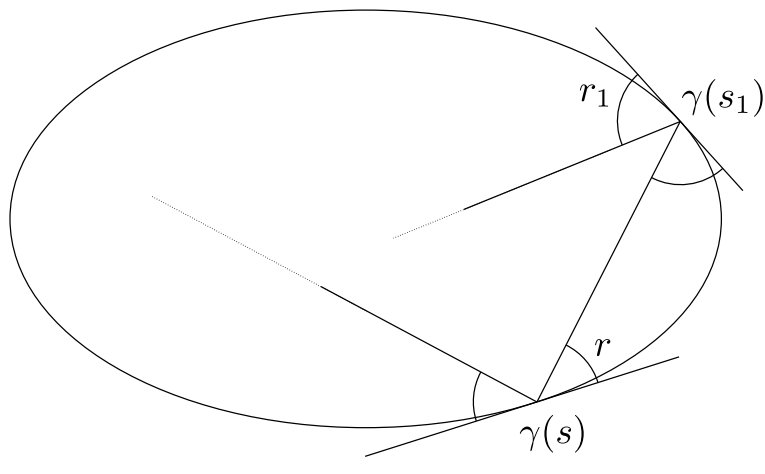

Figure 1: The billiard map $f(s, r)=\left(s_{1}, r_{1}\right)$.

the cylinder in the same way that the orbits of the rigid rotation of angle $p / q$. The Poincaré-Birkhoff Theorem states that there exist at least two different Birkhoff $(p, q)$-periodic orbits $[2,10]$. If $O=\left\{\left(x_{j}, y_{j}\right)\right\}_{j \in \mathbb{Z}}$ is a $(p, q)$-periodic orbit of $f$, then

$$
h\left(x_{j+q}, x_{j+q+1}\right)=h\left(x_{j}+p, x_{j+1}+p\right)=h\left(x_{j}, x_{j+1}\right),
$$

so there are only $q$ different terms in the action functional $W$, which encode the $(p, q)$-periodic dynamics. In particular, any $(p, q)$-periodic orbit $O=\left\{\left(x_{j}, y_{j}\right)\right\}_{j \in \mathbb{Z}}$ is in correspondence with a stationary configuration $\mathbf{x}=\left(x_{0}, \ldots, x_{q-1}\right) \in \mathbb{R}^{q-1}$ of the $(p, q)$-periodic action

$$
W^{(p, q)}[\mathbf{x}]=h\left(x_{0}, x_{1}\right)+h\left(x_{1}, x_{2}\right)+\cdots+h\left(x_{q-1}, x_{0}+p\right) .
$$

We say that $W^{(p, q)}[O]=W^{(p, q)}[\mathbf{x}]$ is the $(p, q)$-periodic action of the $(p, q)$-periodic orbit $O$. The Birkhoff $(p, q)$-periodic orbits provided by the Poincaré-Birkhoff Theorem correspond to the minimizing and minimax stationary configurations of $W^{(p, q)}$.

Mather defined the quantity $\Delta W_{p / q} \geq 0$ as the action of the minimax periodic orbit minus the action of the minimizing one [9]. Mather's $\Delta W_{p / q}$ has a nice dynamical interpretation. It is equal to the flux through any homotopically non trivial curve without self-intersections passing through all the points of both the minimizing and the minimax $(p, q)$-periodic orbits. This is the MacKay-Meiss-Percival action principle [8]. Thus, $\Delta W_{p / q}$ gives a rough estimation of the size of the $(p, q)$ resonance of the twist map. We also recall that the hyperbolic (respectively, elliptic) periodic orbits in a given resonance are generically minimizing (respectively, minimax).

Next, we adapt these ideas to billiard maps.

Let $Q$ be a smooth strictly convex curve in the Euclidean plane. For simplicity, we assume that $l=\operatorname{Length}(Q)=1$. Let $\gamma: \mathbb{T} \rightarrow Q, s \mapsto \gamma(s)$, be an arc-length counterclockwise parametrization of $Q$. The bounce position of the particle inside $Q$ is determined by the arc-length parameter $s$. The direction of motion is measured by the angle of incidence $r \in(0, \pi)$. Let

$$
f: \mathbb{T} \times(0, \pi) \rightarrow \mathbb{T} \times(0, \pi), \quad f(s, r)=\left(s_{1}, r_{1}\right),
$$

be the corresponding billiard map. Figure 1 illustrates this map. The coordinates $(s, r)$ are called Birkhoff coordinates.

Let $\ell\left(s, s_{1}\right)=\left|\gamma(s)-\gamma\left(s_{1}\right)\right|$ be the Euclidean distance between two impact points on $Q$. It is easy to prove that

$$
\frac{\partial \ell}{\partial s}\left(s, s_{1}\right)=-\cos r, \quad \frac{\partial \ell}{\partial s_{1}}\left(s, s_{1}\right)=\cos r_{1} .
$$




\begin{tabular}{crrr}
\hline \hline$(p, q)$ & $H^{(p, q)}$ & $E^{(p, q)}$ & $\Delta W_{p / q}$ \\
\hline$(1,2)$ & 4.000000 & 3.828482 & 0.171577 \\
$(1,4)$ and $(3,4)$ & 5.594652 & 5.536901 & 0.057751 \\
$(1,3)$ and $(2,3)$ & 5.115169 & 5.112940 & 0.002229 \\
$(3,8)$ and $(5,8)$ & 14.773311 & 14.772302 & 0.001009 \\
$(5,12)$ and $(7,12)$ & 23.151909 & 23.150969 & 0.000940 \\
$(3,10)$ and $(7,10)$ & 15.925337 & 15.924445 & 0.000892 \\
$(1,6)$ and $(5,6)$ & 5.904338 & 5.903527 & 0.000811 \\
$(2,5)$ and $(3,5)$ & 9.366997 & 9.366503 & 0.000494 \\
$(1,8)$ and $(7,8)$ & 6.024507 & 6.024232 & 0.000275 \\
$(3,7)$ and $(4,7)$ & 13.455442 & 13.455236 & 0.000206 \\
$(1,5)$ and $(4,5)$ & 5.785133 & 5.785011 & 0.000122 \\
\hline \hline
\end{tabular}

Table 1: The biggest Mather's $\Delta W_{p / q}$ for the billiard inside $x^{2}+y^{2}+y^{4} / 10=1$.

If we consider the coordinates $(x, y)=(s,-\cos r) \in \mathbb{T} \times(-1,1)$, then the billiard map $f$ becomes a smooth exact twist map with Lagrangian $h\left(x, x_{1}\right)=-\ell\left(s, s_{1}\right)$ and boundary rotation numbers $\varrho_{-}=0$ and $\varrho_{+}=1$. That is, the action of a periodic billiard trajectory is, up to the sign, its length. In particular, Mather's $\Delta W_{p / q}$ is the length of the $(p, q)$-periodic billiard trajectory that minimizes the action (and so, maximizes the length) minus the length of the minimax one. Generically,

$$
\Delta W_{p / q}=H^{(p, q)}-E^{(p, q)},
$$

where $H^{(p, q)}$ and $E^{(p, q)}$ are the lengths of the hyperbolic and elliptic $(p, q)$-periodic billiard trajectories inside $Q$. For instance, $H^{(1,2)}=4 a, E^{(1,2)}=4 b$, and $\Delta W_{1 / 2}=4(a-b)$ for the billiard inside the ellipse $x^{2} / a^{2}+y^{2} / b^{2}=1$ with $0<b<a$.

Note that any $(p, q)$-periodic billiard trajectory gives rise to a $(q-p, q)$-periodic one by inverting the direction of motion. This means that $\Delta W_{p / q}=\Delta W_{(q-p) / q}$ for all $p / q \in(0,1 / 2)$.

We have listed the biggest Mather's $\Delta W_{p / q}$ for the billiard inside the perturbed circle $x^{2}+y^{2}+y^{4} / 10=1$. See Table 1 . The rest of Mather's $\Delta W_{p / q}$ are smaller that $10^{-4}$. The values in the table suggest that the $(1,2)$-resonance and both $(p, 4)$ resonances should be the most important ones. This prediction is confirmed in Figure 2, where we display the biggest resonances of the billiard map inside $x^{2}+y^{2}+y^{4} / 10=1$.

Mather's $\Delta W_{p / q}$ allow us to single out the most important resonances, but they do not give an exact measure of the size of resonances. To begin with, there is not a unique way to define such size. A choice is the area $A_{p / q}$ of the Birkhoff instability region that contains the $(p, q)$-resonance. A Birkhoff instability region is a region of the phase space delimited by two rotational invariant curves (RICs) without any other RIC in its interior. If we have a twist map with a $(p, q)$-resonant RIC, then $\Delta W_{p / q}=$ $\mathrm{O}(\epsilon)$ and $A_{p / q}=\mathrm{O}\left(\epsilon^{1 / 2}\right)$ under generic perturbations of order $\mathrm{O}(\epsilon)$. See [37]. This shows up a clear difference between these two quantities. For instance, the billiard map inside the circle $x^{2}+y^{2}=1$ has a $(1,2)$-resonant RIC, which is destroyed under the perturbation $x^{2}+y^{2} /(1-\epsilon)^{2}=1$. However, this perturbed billiard table is integrable (it is an ellipse), so both quantities can be analytically computed: $\Delta W_{1 / 2}=4 \epsilon$ and $A_{1 / 2}=8 \epsilon^{1 / 2}$. We omit the details.

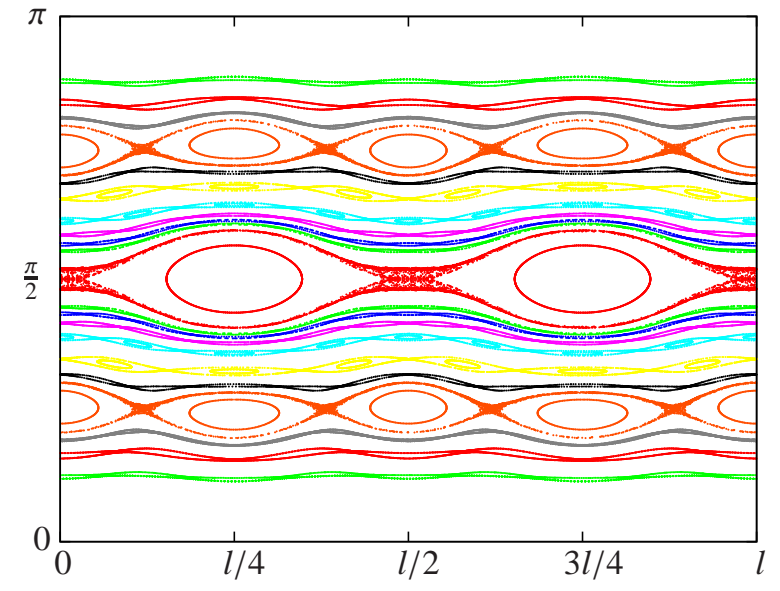

Figure 2: The biggest $(p, q)$-resonances of the billiard map $f(s, r)=\left(s_{1}, r_{1}\right)$ inside the perturbed circle $Q=\left\{(x, y) \in \mathbb{R}^{2}: x^{2}+y^{2}+y^{4} / 10=1\right\}$. We recall that $l=\operatorname{Length}(Q)$. All $(p, q)$-resonances with odd period $q$ have $2 q$ elliptic islands due to the bi-axisymmetric character of the curve. From bottom to top: $(1,8),(1,6),(1,5),(1,4),(3,10),(1,3),(3,8),(2,5),(5,12),(3,7),(1,2)$, and their $(q-p, q)$ symmetric counterparts.

\section{A candidate for limit problem}

To begin with, we recall how to obtain the limit problem for the splitting of separatrices of the generalized standard map $f(x, y)=\left(x_{1}, y_{1}\right)$ given by

$$
x_{1}=x+y_{1}, \quad y_{1}=y+\epsilon p(x) .
$$

For simplicity, we assume that $p(x)$ is a polynomial, $p(0)=0$, and $p^{\prime}(0)=1$, so the origin is a hyperbolic fixed point of $f$ with eigenvalues $\lambda=\mathrm{e}^{h}$ and $\lambda^{-1}=\mathrm{e}^{-h}$, where $\epsilon=4 \sinh ^{2}(h / 2)$. There is numerical evidence that the splitting size in this kind of polynomial standard maps satisfies the asymptotic formula (2) for some exponent $r>0$, some power $m \in \mathbb{R}$, and some constant or oscillating function $A(1 / h)$. We determine the exponent following [23].

First, we transform the original map into the map

$$
x_{1}=x+\mu z_{1}, \quad z_{1}=z+\mu p(x)
$$

by means of the scaling $z=y / \mu$, where $\mu=\sqrt{\epsilon}$. Note that $\mu \asymp h$ as $\epsilon \rightarrow 0^{+}$. The dynamics of this map for small $\mu$ resembles the dynamics of the $\mu$-time flow of the Hamiltonian $H_{0}(x, z)=$ $z^{2} / 2-\int p(x) \mathrm{d} x$. Besides, the origin is a hyperbolic equilibrium point of the Hamiltonian system

$$
x^{\prime}=\partial_{z} H_{0}(x, z)=z, \quad z^{\prime}=-\partial_{x} H_{0}(x, z)=p(x) .
$$

If the singular level set $\left\{(x, z) \in \mathbb{R}^{2}: H_{0}(x, z) \equiv H_{0}(0,0)\right\}$ contains a separatrix to the origin, then we compute the flow on it and we get a homoclinic solution $\left(x_{0}(\xi), z_{0}(\xi)\right)$ that can be seen as the limit of the map on its separatrices when $\epsilon \rightarrow 0^{+}$. Such homoclinic solution is determined, up to a constant time shift, by imposing

$$
x_{0}^{\prime \prime}(\xi)=p\left(x_{0}(\xi)\right), \quad \lim _{\xi \rightarrow \pm \infty} x_{0}(\xi)=0 .
$$


It turns out that there exists $\delta>0$ such that $x_{0}(\xi)$ is analytic in the open complex strip $\mathcal{I}_{\delta}=\{\xi \in \mathbb{C}:|\mathfrak{J} \xi|<\delta\}$ and has singularities on the boundary of $\mathcal{I}_{\delta}$. Then $r=2 \pi \delta$. This claim is contained in [23], although a complete proof is still pending. However, Fontich and Simó proved the following exponentially small upper bound in [18]. If $\alpha \in(0, \delta)$, then there exist some constants $K, h_{0}>0$ such that

$$
\text { "splitting size" } \leq K \mathrm{e}^{-2 \pi \alpha / h}, \quad \forall h \in\left(0, h_{0}\right] .
$$

We want to emphasize an essential, but sometimes forgotten, hypothesis of the Fontich-Simó Theorem. Let

$$
\sigma_{0}(\xi)=\left(x_{0}(\xi), y_{0}(\xi)\right)
$$

be the original homoclinic solution. The generalized standard map (11) should have an analytic extension to a complex neighborhood in $\mathbb{C}^{2}$ of $\sigma_{0}\left(\overline{\mathcal{I}_{\alpha}}\right)$. If $p(x)$ is a polynomial, then $f$ can be extended to the whole $\mathbb{C}^{2}$ and this hypothesis is automatically fulfilled. On the contrary, it remains to be checked when $p: \mathbb{R} \rightarrow \mathbb{R}$ is just a real analytic function.

Next, we adapt these ideas to our billiard problem.

Let $Q$ be an analytic strictly convex curve in the Euclidean plane. Set $l=\operatorname{Length}(Q)$. Let $\kappa(s)$ be the curvature of $Q$ in some arc-length parameter $s \in \mathbb{R} / l \mathbb{Z}$. Note that $\kappa(s)>0$ for all $s \in \mathbb{R} / l \mathbb{Z}$. Let $\rho(s)=1 / \kappa(s)$ be the radius of curvature. We are interested in the dynamics of the billiard map (10) when the angle of incidence $r$ tends to zero. More precisely, we consider that $r=\mathrm{O}(1 / q)$ and $q \rightarrow+\infty$.

Lazutkin [36] gave the Taylor expansion

$$
s_{1}=s+2 \varrho(s) r+\mathrm{O}\left(r^{2}\right), \quad r_{1}=r-2 \varrho^{\prime}(s) r^{2} / 3+\mathrm{O}\left(r^{3}\right)
$$

for the dynamics of the billiard map (10) around $r=0$. Once fixed a period $q \gg 1$, we take $\mu=1 / q \ll 1$ as the small parameter. Then we transform the previous expansion into

$$
s_{1}=s+\mu \varrho(s) v^{1 / 2}+\mathrm{O}\left(\mu^{2}\right), \quad v_{1}=v-\frac{2}{3} \mu \varrho^{\prime}(s) v^{3 / 2}+\mathrm{O}\left(\mu^{2}\right),
$$

by means of the change of variables $\sqrt{v}=2 r / \mu$. The billiard dynamics for small $\mu$ resembles the dynamics of the $\mu$-flow of the Hamiltonian $H_{0}(s, v)=\frac{2}{3} \varrho(s) v^{3 / 2}$. That is, the $\mu$-flow of the Hamiltonian system

$$
s^{\prime}=\varrho(s) v^{1 / 2}, \quad v^{\prime}=-\frac{2}{3} \varrho^{\prime}(s) v^{3 / 2} .
$$

We compute the flow on the level set $\mathcal{H}_{C}:=\left\{H_{0}(s, v) \equiv \frac{2}{3} C^{3}\right\}$, for some constant $C>0$. If $(s, v) \in \mathcal{H}_{C}$, then the first equation of the Hamiltonian system reads as

$$
\frac{\mathrm{d} s}{\mathrm{~d} \xi}=s^{\prime}=\varrho(s) v^{1 / 2}=C \varrho^{2 / 3}(s),
$$

or, equivalently, as

$$
C \frac{\mathrm{d} \xi}{\mathrm{d} s}=\kappa^{2 / 3}(s)
$$

We only need the following observations to determine $C$. We are looking at the $(1, q)$-periodic trajectories inside $Q$. We have approximated the billiard dynamics by the $\mu$-time of the Hamiltonian flow with $\mu=1 / q$. Any $(1, q)$-periodic trajectory gives one turn after $q$ iterates of the billiard map, so the variable $\xi$ should be increased by one if $s$ is increased by $l=\operatorname{Length}(Q)$. Therefore,

$$
C=C \int_{0}^{l} \frac{\mathrm{d} \xi}{\mathrm{d} s} \mathrm{~d} s=\int_{0}^{l} \kappa^{2 / 3}(s) \mathrm{d} s=\int_{Q} \kappa^{2 / 3} \mathrm{~d} s .
$$

Relation (9) is obtained by joining equations (12) and (13). Let $s=s_{0}(\xi)$ be the inverse of the solution $\xi=\xi_{0}(s)$ of the differential equation (9) determined, for the sake of definiteness, by the initial condition $\xi_{0}(0)=0$. By abusing the notation, let $\kappa(\xi)=\kappa\left(s_{0}(\xi)\right)$ be the curvature in the new angular variable $\xi \in \mathbb{R} / \mathbb{Z}$. Then $\kappa(\xi)$ is a 1 -periodic real analytic function which does not vanish on the reals. Let us assume that there exists $\delta>0$ such that $\kappa(\xi)$ is analytic and does not vanish on the open complex strip $\mathcal{I}_{\delta}$ and has singularities and/or zeros on the boundary of $\mathcal{I}_{\delta}$. Note that we are avoiding not only singularities but also zeros of the curvature $\kappa(\xi)$. On the one hand, the results found by Marvizi and Melrose only hold for smooth strictly convex curves, so the zeros of the curvature are a source of potential problems. On the other hand, several positive and negative fractional powers of the curvature appear in the previous computations (see also below), and such powers are not analytic at the zeros of the curvature.

Following the numerical evidences in the splitting problems of the polynomial standard maps, we thought that $r=2 \pi \delta$, but our experiments disprove it. We have obtained that $r \leq 2 \pi \delta$, the equality being an infrequent situation.

An explanation of such discrepancy is the following one. Set $\sigma_{0}(\xi)=\left(s_{0}(\xi), r_{0}(\xi)\right), r_{0}(\xi)=\mu \sqrt{v_{0}(\xi)} / 2=C \kappa^{1 / 3}(\xi) / 2 q$. We know that the billiard map (10) can be analytically extended to $(\mathbb{R} / l \mathbb{Z}) \times[0, \pi)$; see [14, Proposition 5]. However, we do not know whether it can be analytically extended to a complex neighborhood in $(\mathbb{C} / l \mathbb{Z}) \times \mathbb{C}$ of $\sigma_{0}\left(\overline{\mathcal{I}_{\alpha}}\right)$ as $\alpha \rightarrow \delta^{-}$and $q \rightarrow+\infty$ or not. Hence, the inequality $r \leq 2 \pi \delta$ does not look so bad in the light of the previous discussion about the Fontich-Simó Theorem. In fact, it is commonly accepted that the magnitude involved in the exponent of the exponentially small formulas for splitting problems is not the minimum distance to the real line of the set of singularities of the time parametrization of the separatrix but the minimum distance to the real line of the set of singularities of the perturbation of the system when evaluated on the time parametrization of the separatrix. See [29, 38] for some examples. It seems reasonable to think that one has to compute the singularities of the Lagrangian evaluated on the solution of (9), which, in its turn, reduces to the study of the singularities of $\gamma\left(s_{0}(\xi)\right)$. This is a work in progress.

\section{Model tables}

We restrict our study to the perturbed ellipses and perturbed circles given implicitly in (7). To be precise, the algebraic curve $x^{2}+y^{2} / b^{2}+\epsilon y^{n}=1$ has several real connected components when $n$ is odd. Henceforth, we only consider the one that tends to the ellipse (or circle) $x^{2}+y^{2} / b^{2}=1$ as $\epsilon$ tends to zero. 
Let $\epsilon_{n}=\epsilon_{n}(b)$ be the maximal positive parameter such that

$Q$ is analytic and strictly convex for all $\epsilon \in I_{n}:=\left(0, \epsilon_{n}\right)$.

On the one hand, $I_{n}=(0,+\infty)$ when $n$ is even. In such cases, we will reach the value $\epsilon=1$ in some numerical computations. On the other hand, if $n$ is odd, the algebraic curve defined by $x^{2}+y^{2} / b^{2}+\epsilon y^{n}=1$ has a singular point on the $y$-axis when

$$
\epsilon=\bar{\epsilon}_{n}=\bar{\epsilon}_{n}(b):=2(n-2)^{n / 2-1} n^{-n / 2} b^{-n} .
$$

Thus, $Q$ is no longer analytic when $\epsilon=\bar{\epsilon}_{n}$. Our computations suggest that $\epsilon_{n}=\bar{\epsilon}_{n}$ so we restrict our experiments to the range $0<\epsilon<\bar{\epsilon}_{n}$. We note that $\bar{\epsilon}_{3}(b) \approx 0.3849 / b^{3}, \bar{\epsilon}_{5}(b) \approx 0.1859 / b^{5}$, and $\bar{\epsilon}_{7}(b) \approx 0.1232 / b^{7}$. We also restrict our experiments to the degrees $3 \leq n \leq 8$.

The symmetries of our model tables simplify the search of some periodic trajectories. If $n$ is even, $Q$ is symmetric with respect to both axis of coordinates, so $Q$ is bi-axisymmetric. If $n$ is odd, $Q$ is symmetric with respect to the $y$-axis only, so $Q$ is axisymmetric but not bi-axisymmetric. We say that a billiard trajectory is axisymmetric when its corresponding polygon is symmetric with respect to some axis of coordinates. We only compute axisymmetric periodic trajectories, APTs for short.

First, let us focus on the case odd $n$. The axisymmetric trajectories inside $Q$ are characterized as the ones with an impact point on or with a segment perpendicular to the $y$-axis. The APTs are characterized as the ones satisfying twice the former condition. Thus, there are four kinds of APTs inside $Q$. Besides, only two of these kinds are possible depending on the (parity of the) period $q$.

The classification for even $n$ is richer because the symmetry with respect to the $x$-axis plays the same role. See Table 2.

We wanted to study the differences $\Delta^{(1, q)}$ and the Mather's $\Delta W_{1 / q}$, but instead we will compute the signed differences $D_{q}$ between the lengths of the $(1, q)$-APTs. Clearly, $\left|D_{q}\right| \leq \Delta^{(1, q)}$. In some cases, all periodic trajectories are axisymmetric, and so $\Delta^{(1, q)}=\Delta W_{1 / q}=\left|D_{q}\right|$. See Proposition 2 .

We will fix the semi-minor axis $b$ and the degree $n$ in our numerical experiments. That is, we will study the dependence of $D_{q}=D_{q}(\epsilon)$ on the perturbative parameter $\epsilon$ and the period $q$. The quantity $D_{q}(\epsilon)$ is analytic at $\epsilon=0$ because all $(1, q)$-APTs are so. On the contrary, the period $q$ is a singular parameter of this problem because $D_{q}$ is exponentially small in $q$. Thus, we will deal with:

- The regular case, where we study the asymptotic behavior of $D_{q}(\epsilon)$ when $\epsilon \rightarrow 0$ and $q \geq 3$ is fixed; and

- The singular case, where we study the asymptotic behavior of $D_{q}(\epsilon)$ when $q \rightarrow+\infty$ and $\epsilon \in \mathbb{R}$ is fixed.

We will see that the classical Melnikov method is suitable to study the regular case but it is not so to study the singular one. Besides, the Melnikov method gives more information on perturbed ellipses than on perturbed circles. The singular case is only studied numerically.

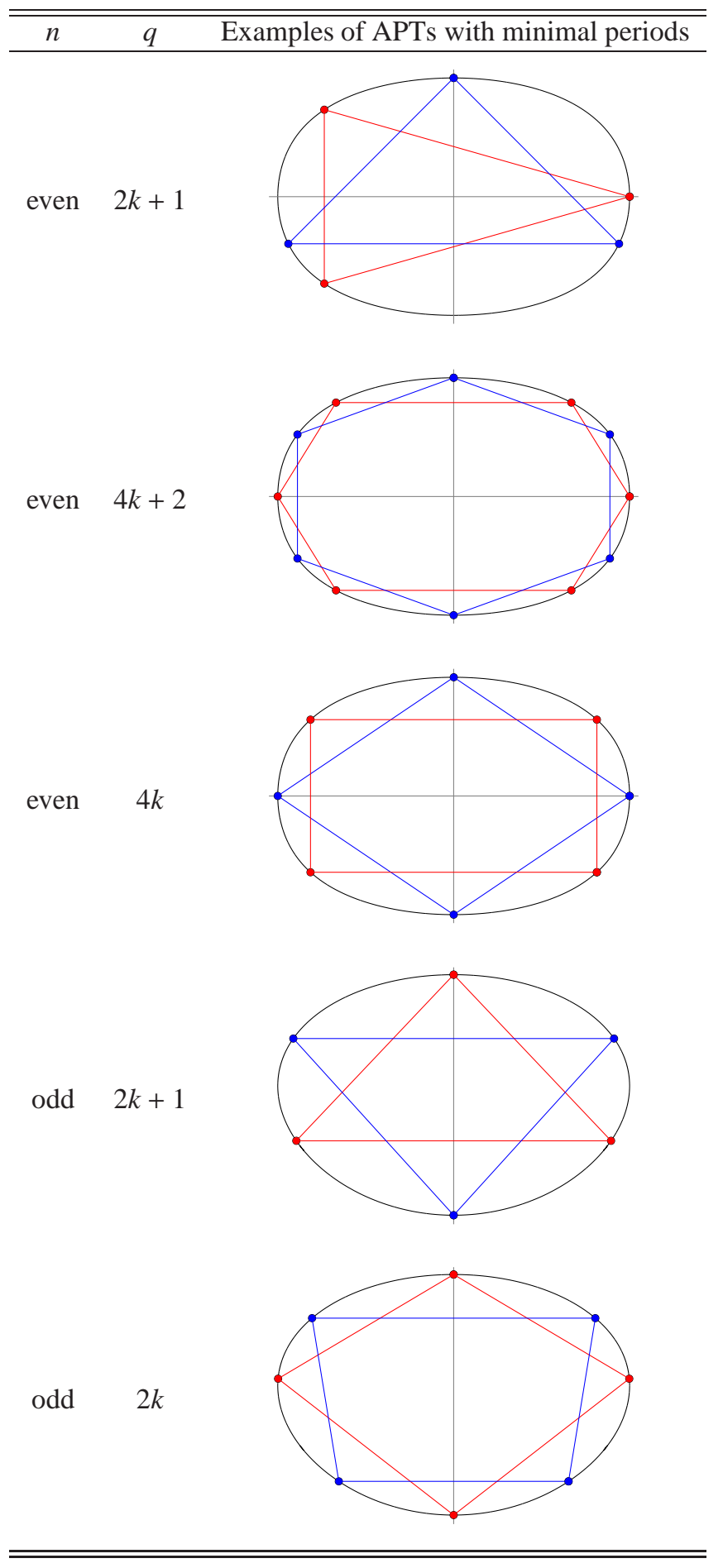

Table 2: Classification of $(1, q)$-APTs inside bi-axisymmetric and axisymmetric billiard tables $Q$. In each case, the difference $D_{q}$ is the length of the $(1, q)$-APT in red minus the length of the $(1, q)$-APT in blue. The gray lines denote the axis of symmetry.

\section{Perturbed ellipses}

In this section we restrict ourselves to the case $0<b<1$. We begin with the regular case, so the semi-minor axis $b$, the degree $n \geq 3$, and the period $q \geq 3$ are fixed, whereas $\epsilon \rightarrow 0^{+}$. 
We will check that

$$
\Delta^{(1, q)}=\Delta^{(1, q)}(\epsilon)=\epsilon \Delta_{1}^{(1, q)}+\mathrm{o}(\epsilon),
$$

for some coefficient $\Delta_{1}^{(1, q)} \in \mathbb{R}$. This coefficient can be computed by using a standard Melnikov method. In fact, the model tables (7) have been chosen in such a way that the asymptotic behavior of $\Delta_{1}^{(1, q)}$ can be determined. The analytical results for $\Delta_{1}^{(1, q)}$ in the cubic and quartic perturbations are stated below, but we need to introduce some notation first.

Given $m \in[0,1)$, the complete elliptic integral of the first kind is

$$
K=K(m)=\int_{0}^{\pi / 2}\left(1-m \sin ^{2} \theta\right)^{-1 / 2} \mathrm{~d} \theta .
$$

We also write $K^{\prime}=K^{\prime}(m)=K(1-m)$.

Proposition 2. If $b \in(0,1)$ and $q \geq 3$, the following properties hold.

1. $\Delta_{1}^{(1, q)}=0$, for odd $n$ and even $q$.

2. There exist some constants $c, M_{3}, M_{4}, K_{4}>0$, depending only on $b$, such that

$$
\Delta_{1}^{(1, q)} \asymp \begin{cases}M_{3} \mathrm{e}^{-c q}, & \text { for } n=3 \text { and odd } q, \\ K_{4} q \mathrm{e}^{-2 c q}, & \text { for } n=4 \text { and odd } q, \\ M_{4} q \mathrm{e}^{-c q}, & \text { for } n=4 \text { and even } q,\end{cases}
$$

when $q \rightarrow+\infty$. Besides, $K_{4}=2 M_{4}$, and

$$
c=\frac{\pi K\left(b^{2}\right)}{2 K\left(1-b^{2}\right)}=\frac{\pi K^{\prime}\left(1-b^{2}\right)}{2 K\left(1-b^{2}\right)} .
$$

3. If $n=3$ and $q$ is odd or if $n=4$, then there exists $\tilde{\epsilon}_{n}=\tilde{\epsilon}_{n}(b, q) \in I_{n}$ such that all $(1, q)$-periodic billiard trajectories inside (7) are axisymmetric when $\epsilon \in\left(0, \tilde{\epsilon}_{n}\right)$. In particular, $\Delta^{(1, q)}=\Delta W_{1 / q}=\left|D_{q}\right|$ for all $\epsilon \in\left(0, \tilde{\epsilon}_{n}\right)$.

See Appendix A for the proof. The explicit values of $M_{3}$ and $M_{4}$ can be found in (A.7). Related computations can be found in [34].

Remark 1. Similar results hold for any degree $n \geq 5$, although it is more cumbersome to compute the Melnikov constants $M_{n}$ (and $K_{n}$ if $n$ is even) and the Melnikov powers $m_{n}$ such that

$$
\Delta_{1}^{(1, q)} \asymp \begin{cases}M_{n} q^{m_{n}} \mathrm{e}^{-c q}, & \text { for odd } n \text { and odd } q, \\ K_{n} q^{m_{n}} \mathrm{e}^{-2 c q}, & \text { for even } n \text { and odd } q, \\ M_{n} q^{m_{n}} \mathrm{e}^{-c q}, & \text { for even } n \text { and even } q,\end{cases}
$$

as $q \rightarrow+\infty$. The Melnikov exponent $c$ does not depend on $n$.

From the first order formula (16), we deduce that

$$
\lim _{\epsilon \rightarrow 0}\left[\Delta^{(1, q)} / \epsilon \Delta_{1}^{(1, q)}\right]=1
$$

for any fixed $q \geq 3$. Next, we wonder whether the roles of $\epsilon$ and $q$ are interchangeable; that is, if

$$
\lim _{q \rightarrow+\infty}\left[\Delta^{(1, q)} / \epsilon \Delta_{1}^{(1, q)}\right]=1,
$$

for any fixed but small enough $\epsilon>0$.

We should compute $\Delta^{(1, q)} / \epsilon \Delta_{1}^{(1, q)}$ for big periods $q$ in order to answer this question, but instead we compute $\left|D_{q}\right| / \epsilon \Delta_{1}^{(1, q)}$. Both quotients coincide if $\epsilon$ is small enough, see Proposition 2. We do not compute $\left|D_{q}\right| / \epsilon \Delta_{1}^{(1, q)}$ for the cubic perturbation and even periods, because $\Delta_{1}^{(1, q)}=0$ for $n=3$ and even $q$.

We show the results obtained for the cubic and quartic perturbations in Figure 3. These figures are obtained by taking the semi-minor axis $b=4 / 5$. Other values for the semi-minor axis give rise to similar figures.

The Melnikov method does not predict the asymptotic behavior of $\Delta^{(1, q)}$ in the singular case. That is, limit (19) does not hold. Indeed, if we fix any $\epsilon>0$, then the quotient $\left|D_{q}\right| / \epsilon \Delta_{1}^{(1, q)}$ drifts away from one as $q$ grows. The drift appears earlier for odd periods in the case of the quartic perturbation. As $\epsilon$ gets smaller, the drift appears at larger periods $q$. Since the computing time grows quickly when $q$ grows, the computations to see that drift when $\epsilon$ is very small are unfeasible with our resources. This happens, for instance, when $n=4$ and $\epsilon=10^{-30}$. See Figure 3.

Based on these numerical experiments, we guess that there exist some critical exponents $v_{n}>0$ such that

$$
\Delta^{(1, q)}=\Delta^{(1, q)}(\epsilon) \asymp \begin{cases}M_{n} \epsilon q^{m_{n}} \mathrm{e}^{-c q}, & \text { for odd } n \text { and odd } q, \\ K_{n} \epsilon q^{m_{n}} \mathrm{e}^{-2 c q}, & \text { for even } n \text { and odd } q, \\ M_{n} \epsilon q^{m_{n}} \mathrm{e}^{-c q}, & \text { for even } n \text { and even } q,\end{cases}
$$

when $\epsilon=\mathrm{O}\left(q^{-v}\right), q \rightarrow+\infty$, and $v>v_{n}$. Here, $M_{n}, K_{n}, m_{n}$, and $c$ are the Melnikov quantities introduced in Proposition 2 and Remark 1. We do not give an asymptotic behavior when $n$ is odd and $q$ is even because we do not have any Melnikov prediction for that case. Results about exponentially small asymptotic behaviors based on Melnikov predictions are common in the literature. For instance, the rapidly forced pendulum is studied in [23, 38-41] and some perturbed McMillan maps are studied in $[20,27,28,31]$.

Nevertheless, we are interested in a more natural problem. Namely, the asymptotic behavior of $\Delta^{(1, q)}$ when $q \rightarrow+\infty$ and $\epsilon$ is fixed. As we have said before, we compute the signed difference $D_{q}$ instead of $\Delta^{(1, q)}$. We have numerically checked that, if $\epsilon$ is small enough, then there exist a constant $A \neq 0$, a power $m \in \mathbb{Z}$, and an exponent $r>0$ such that

$$
D_{q} \asymp A q^{m} \mathrm{e}^{-r q},
$$

as $q \rightarrow+\infty$. In fact, the real behavior is slightly more complicated, since these three quantities depend on the parity of $q$. We summarize our results as follows.

Numerical Result 3. Fix $b \in(0,1)$ and $n \geq 3$. Let $I_{n}$ be the maximal interval defined in (14). There exists $\hat{\epsilon}_{n}=\hat{\epsilon}_{n}(b) \in I_{n}$ such that the billiard inside (7) satisfies the following properties for all $\epsilon \in\left(0, \hat{\epsilon}_{n}\right)$. The Borel transform (4) has a radius of convergence $\rho \in(0,+\infty)$. Set $r=\rho / 2$. There exist two constants $A, B \neq 0$ such that

$$
D_{q} \asymp \begin{cases}B q^{-2} \mathrm{e}^{-2 r q}, & \text { for even } n \text { and odd } q, \\ A q^{-3} \mathrm{e}^{-r q}, & \text { otherwise, }\end{cases}
$$




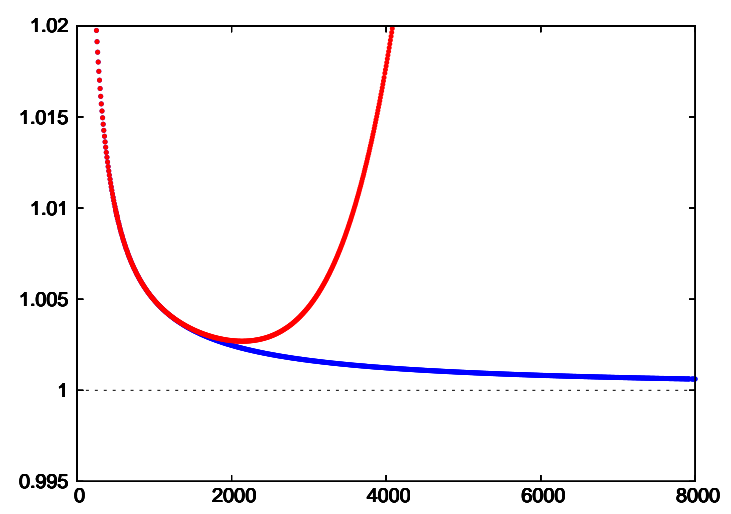

(a) $n=3$ and odd periods. Red: $\epsilon=10^{-10}$. Blue: $\epsilon=10^{-30}$.

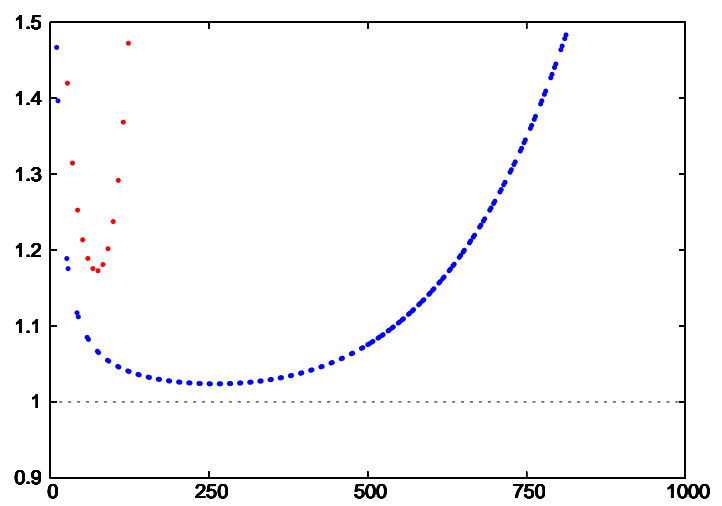

(b) $n=4$ and $\epsilon=10^{-10}$. Red: odd periods. Blue: even periods.

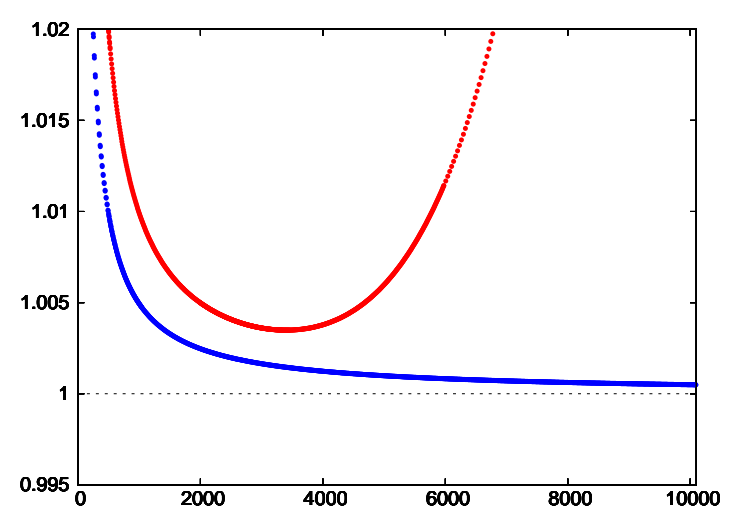

(c) $n=4$ and $\epsilon=10^{-20}$. Red: odd periods. Blue: even periods.

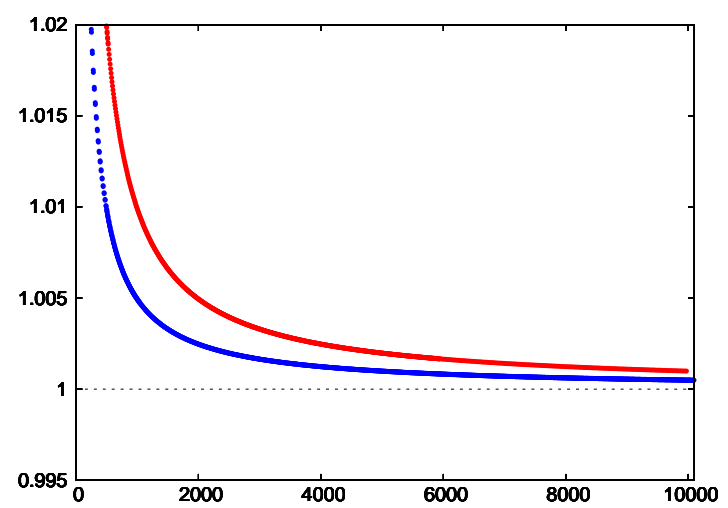

(d) $n=4$ and $\epsilon=10^{-30}$. Red: odd periods. Blue: even periods.

Figure 3: The quotient $\left|D_{q}\right| / \epsilon \Delta_{1}^{(1, q)}$ versus the period $q$ for $b=4 / 5$.

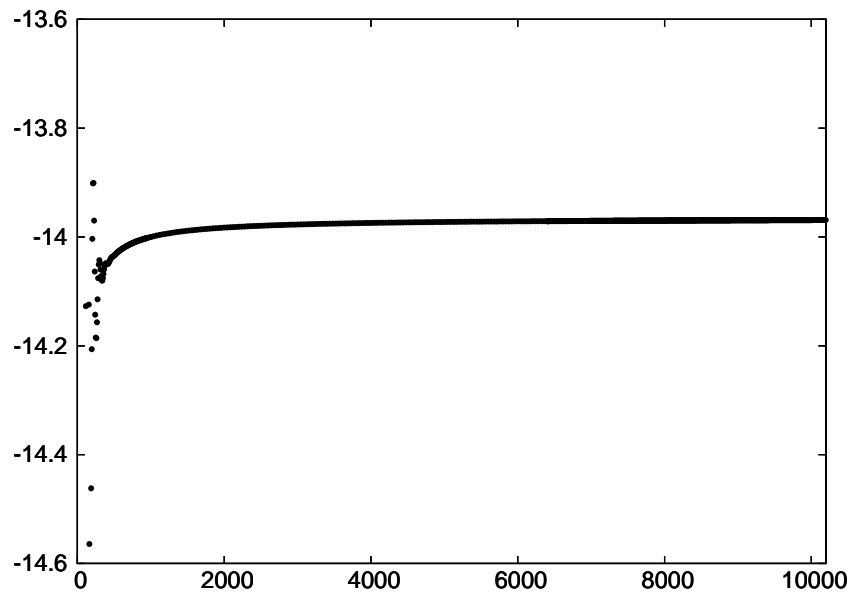

(a) $n=3, b=9 / 10$, and $\epsilon=1 / 10$

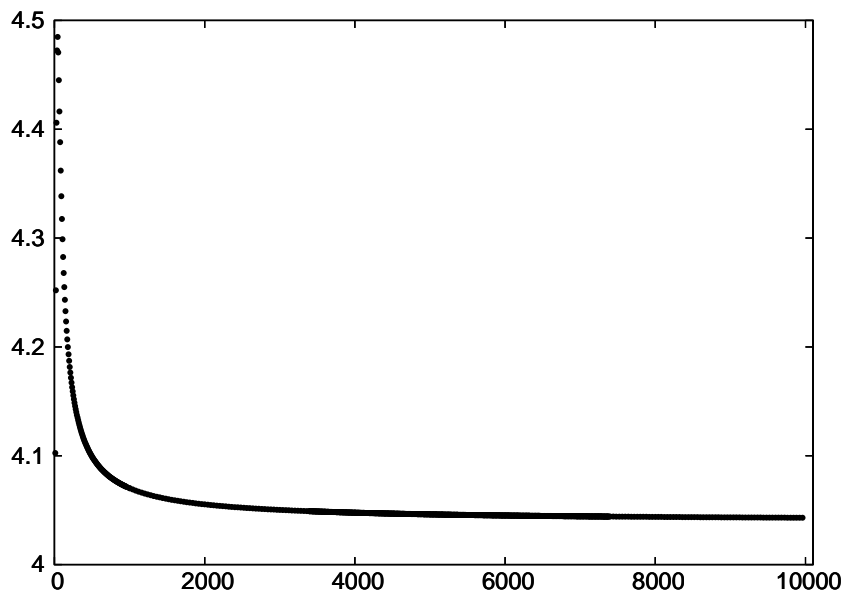

(b) $n=4, b=9 / 10, \epsilon=1 / 10$, and odd periods.

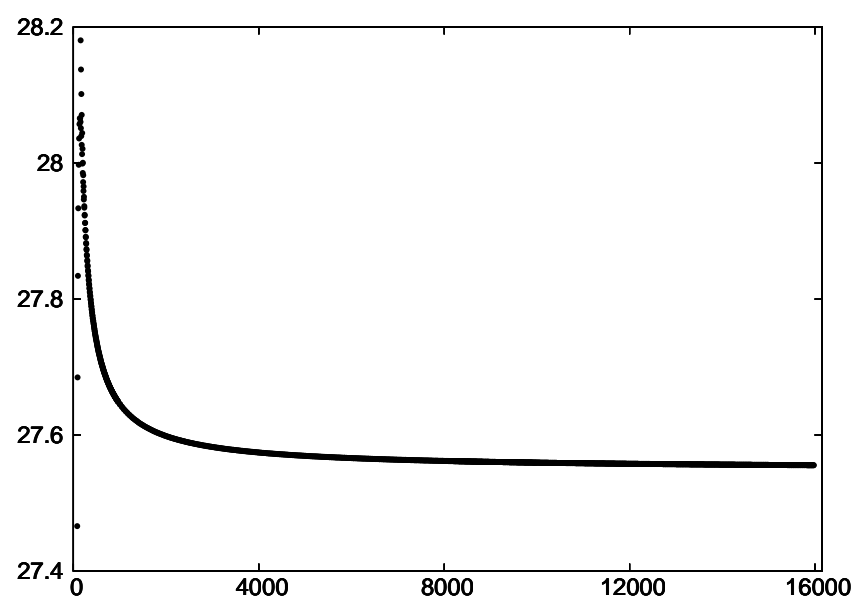

(c) $n=4, b=9 / 10, \epsilon=1 / 10$, and even periods.

Figure 4: The normalized differences $\hat{D}_{q}$ tend to a constant when $q \rightarrow+\infty$ in the ranges $1 / 2 \leq b \leq 9 / 10$ and $0<\epsilon \leq 1 / 10$ for the cubic and quartic perturbations. If $n$ is even, then we have to study the even and odd periods separately. 
as $q \rightarrow+\infty$. The quantities $\rho, r, A$, and $B$ depend on $b, \epsilon$, and $n$. The constant $B$ is defined only when $n$ is even. Besides, $\lim _{\epsilon \rightarrow 0} r=c$, where $c$ is the Melnikov exponent defined in (18).

We stated in Conjecture 1 that the function $A(q)$ that appears in the exponentially small asymptotic formula (6) is constant when the billiard table belongs to a certain open set of the space of axisymmetric algebraic curves. Thus, the previous numerical result fits perfectly into the conjecture.

It is interesting to compare the Melnikov formulas (17) with the asymptotic formulas (21). The asymptotic behavior of $D_{q}$ does not depend on the parity of $q$ when $n$ is odd. The exponents $c$ and $r$ play the same role. Finally, the factors $q^{-2}$ and $q^{-3}$ in (21) can not be directly guessed from the Melnikov formulas.

Let us describe our numerical experiments. First, once the exponent $r$ is determined (see next paragraph), we compute the normalized differences

$$
\hat{D}_{q}= \begin{cases}q^{2} \mathrm{e}^{2 r q} D_{q}, & \text { for even } n \text { and odd } q, \\ q^{3} \mathrm{e}^{r q} D_{q}, & \text { otherwise. }\end{cases}
$$

We have checked that these normalized differences $\hat{D}_{q}$ tend to some constant as $q \rightarrow+\infty$ in the ranges $1 / 2 \leq b \leq 9 / 10$ and $0<\epsilon \leq 1 / 10$. Figure 4 shows this behavior on three different scenarios for $b=9 / 10$ and $\epsilon=1 / 10$.

Let us explain how to compute the exponent $r=r(b, \epsilon, n)$. First, we assume that the exponentially small asymptotic formula (20) can be refined as

$$
D_{q} \asymp q^{m} \mathrm{e}^{-r q} \sum_{j \geq 0} d_{j} q^{-2 j},
$$

for some asymptotic coefficients $d_{j} \in \mathbb{R}$ with $d_{0}=A \neq 0$. This assumption is based on similar refined asymptotic formulas for the splitting of separatrices of analytic maps [22, 27]. By taking logarithms, we find the asymptotic expansion

$$
\frac{1}{q} \log \left(q^{-m} D_{q}\right) \asymp-r+\frac{1}{q} \log \left(\sum_{j \geq 0} \frac{d_{j}}{q^{2 j}}\right) \asymp-r+\sum_{j \geq 0} \frac{\alpha_{j}}{q^{2 j+1}},
$$

for some coefficients $\alpha_{j} \in \mathbb{R}$. Therefore, we can compute $r$ by using a Neville extrapolation method from a sequence of differences $D_{q}$. The longer the sequence, the more correct digits in $r$. We obtain 15 correct digits with the following choices. We fix the perturbed ellipse $Q$, that is, we fix $b \in(0,1), \in \in \mathbb{R}$, and $n \geq 3$. Second, we fix the class of periods $q$, so that we are on one of the cases of Table 2. That is, $q=q(k)=2 k+1$, $q=q(k)=4 k+2, q=q(k)=4 k$, or $q=q(k)=2 k$. Then, we compute $D_{q}$ with at least 400 correct digits on an increasing sequence of 500 periods $q_{i}=q\left(k_{i}\right)$, with $k_{i}=k_{0}+10 i$. The initial period $q_{0}$ is chosen to be big enough so that $\left|D_{q_{0}}\right| \leq 10^{-3000}$. In fact, we perform the Neville extrapolation with two different sequences of 500 periods each which allows us to determine the number of correct digits in the final result. The power $m \in$ $\{-2,-3\}$ is found by trial-and-error.

In Figure 5, we display the exponent $r=r(\epsilon)$ for several values of $b$ for the cubic and quartic perturbations. We also

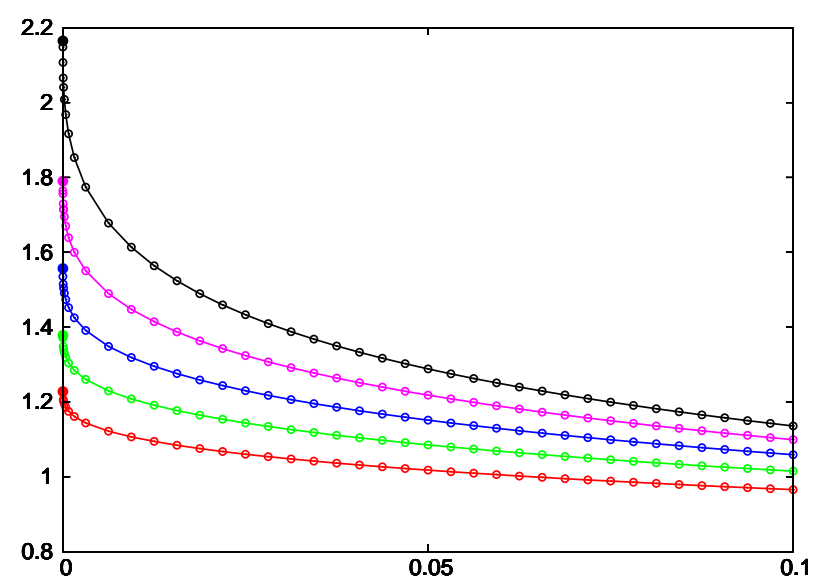

(a) $n=3$.

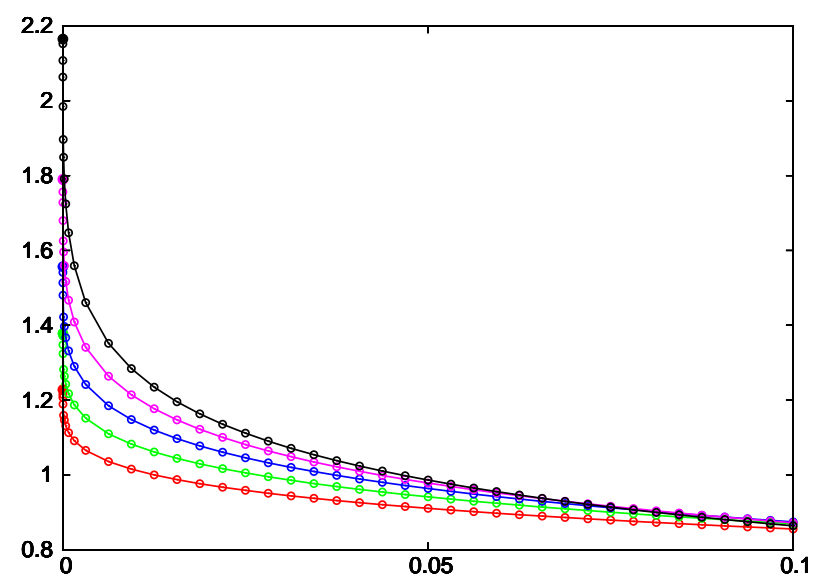

(b) $n=4$.

Figure 5: The exponent $r$ versus the perturbative parameter $\epsilon$. We also display the points $(0, c)$ in solid circles, where $c$ is the Melnikov exponent. We note that $\lim _{\epsilon \rightarrow 0^{+}} r=c$. Red: $b=1 / 2$. Green: $b=3 / 5$. Blue: $b=7 / 10$. Magenta: $b=4 / 5$. Black: $b=9 / 10$.

depict the Melnikov exponent $c$ at $\epsilon=0$ in full circles. Note that $\lim _{\epsilon \rightarrow 0} r=c$ and $r$ is decreasing in $\epsilon$.

Next, let us relate the exponent $r$ with the radius of convergence $\rho$ of the Borel transform (4). Once fixed $b \in(0,1), \epsilon \in \mathbb{R}$, and $n \geq 3$, we compute $\rho=\rho(b, \epsilon, n)$ as follows.

First, we compute the length $L^{(1, q)}$ of one of the $(1, q)$-APTs inside $Q$ for the same sequences of periods $\left(q_{i}\right)$ used for computing $D_{q}$. We use a precision of 3000 correct digits in these computations. The choice of the APT does not matter, since $\left|D_{q_{i}}\right| \leq 10^{-3000}$ for any period $q_{i} \geq q_{0}$. Second, we obtain the first asymptotic coefficients $l_{j}$ in the expansion (1) by using the Neville extrapolation method again. Third, we determine the number of correct digits in each coefficient $l_{j}$ by comparing the results obtained with two different sequences of periods. The number of correct digits in $l_{j}$ decreases as $j$ grows. We always get at least 1500 correct digits in $l_{0}$ and at least 40 correct digits in $l_{450}$.

It turns out that the coefficients $l_{j}$ increase at a factorial rate, so the asymptotic series (1) is Gevrey-1 and diverges for any $q$. Indeed, we have found that there exist a radius of convergence 


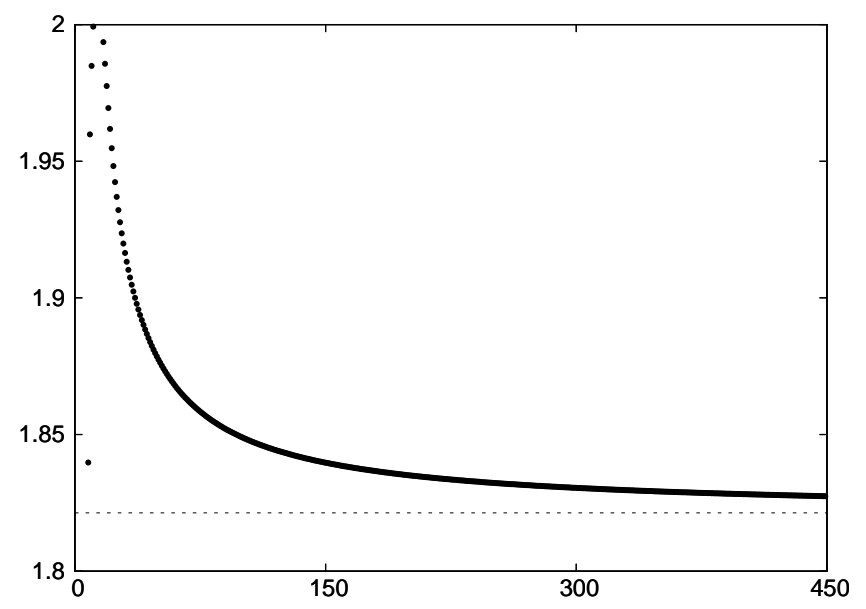

Figure 6: $\left|\hat{l}_{j} / \hat{l}_{j+1}\right|^{1 / 2}$ versus $j$ for $b=9 / 10, \epsilon=1 / 20$, and $n=4$. The dashed line corresponds to the limit value $\rho$ obtained by extrapolation.

$\rho=\rho(b, \epsilon, n)>0$ and a constant $\gamma=\gamma(b, \epsilon, n)>0$ such that

$$
\hat{l}_{j} \asymp \gamma j^{-2} \rho^{-2 j}, \quad j \rightarrow+\infty,
$$

provided $\epsilon$ is small enough. That is, the Borel transform (4) has a singularity at $z=\rho$. In particular,

$$
\rho=\lim _{j \rightarrow \infty}\left|\hat{l}_{j} / \hat{l}_{j+1}\right|^{1 / 2} .
$$

We see this asymptotic behavior in Figure 6.

The rough approximation

$$
\rho \approx\left|\hat{l}_{449} / \hat{l}_{450}\right|^{1 / 2}
$$

only gives about 3 correct digits. If we use an extrapolation method based on the asymptotic expansion

$$
\left|\hat{l}_{j} / \hat{l}_{j+1}\right|^{1 / 2} \asymp \rho+\sum_{i>0} \beta_{i} j^{-i}
$$

the radius of convergence is improved up to 8 correct digits. This is the limit value plotted in Figure 6. We stress that this asymptotic expansion in powers of $j^{-1}$ is probably wrong since the extrapolation becomes unstable after a few steps.

The radius of convergence $\rho$ does not depend on the parity of the periods of the sequence $\left(q_{i}\right)$. Thus, the value of $\rho$ obtained by sequences of different parities must coincide. This provides another validation to the number of correct digits of $\rho$.

Remark 2. Taking into account relation $r=\rho / 2$, we have two different ways of computing the exponent $r$, the direct method and the Borel one. The Borel method is computationally much cheaper. Indeed, the precision required to compute the differences $D_{q_{i}}$ increases along the periods $q_{i}$ whereas it is fixed when computing the lengths $L^{\left(1, q_{i}\right)}$.

At this point, we have established the relations among the Melnikov exponent $c$, the exponent $r$, and the radius of convergence $\rho$. Next, we relate $c$ with the distance $\delta$ provided by our candidate for limit problem, since we are only able to analytically compute $\delta$ for unperturbed ellipses.

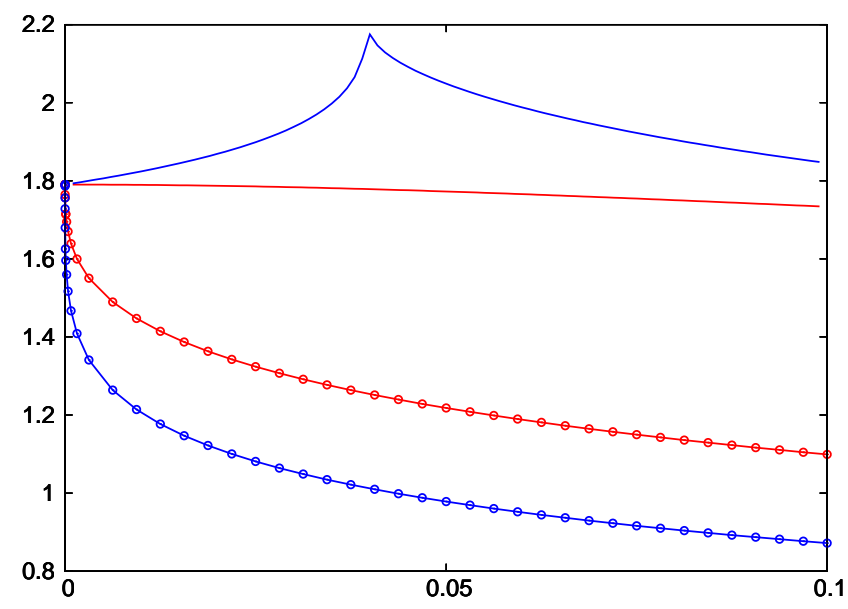

Figure 7: The exponent $r$ (continuous lines with points) and the quantity $2 \pi \delta$ (continuous lines) versus $\epsilon$ for $b=4 / 5$. Red: $n=3$. Blue: $n=4$.

Proposition 4. Let $b \in(0,1)$. Let $\kappa(s)$ be the curvature of the unperturbed ellipse $E=\left\{(x, y) \in \mathbb{R}^{2}: x^{2}+y^{2} / b^{2}=1\right\}$ in some arc-length parameter $s$. Let $\xi \in \mathbb{R} / \mathbb{Z}$ be the angular variable defined by (9). Let $\delta$ be the distance of the set of singularities and zeros of the curvature $\kappa(\xi)$ to the real axis. Then $2 \pi \delta=c$, where $c$ is the Melnikov exponent defined in (18).

This proposition is proved in Appendix B.

We have numerically checked that the inequality $r<2 \pi \delta$ holds in the ranges $1 / 2 \leq b \leq 9 / 10$ and $0<\epsilon \leq 1 / 10$ for the cubic and quartic perturbations. The case $b=4 / 5$ is displayed in Figure 7.

Remark 3. The distance $\delta$ is numerically computed as follows. First, we write the curvature $\kappa$ and the length element $\mathrm{d} s$ of the perturbed ellipse (7) in terms of the vertical coordinate $y$. It turns out that there exist three polynomials $r(y), p(y)$ and $q(y)$ such that

$$
\kappa^{2 / 3} \mathrm{~d} s=g(y) \mathrm{d} y:=\frac{p^{2 / 3}(y)}{\sqrt{r(y) q(y)}} \mathrm{d} y .
$$

For instance, $r(y)=1-y^{2} / b^{2}-\epsilon y^{n}$ and $\operatorname{deg}[p]=\operatorname{deg}[q]=$ $2 n-2$. Let $y_{ \pm}$be the roots of $r(y)$ that tend to $\pm b$ when $\epsilon \rightarrow 0$. The points $\left(0, y_{ \pm}\right)$are the vertices on the vertical axis of the perturbed ellipse (7). Then $\delta=\left|\mathfrak{J} \xi_{\star}\right| / C$, where

$$
C=\int_{Q} \kappa^{2 / 3} \mathrm{~d} s=2 \int_{y_{-}}^{y_{+}} g(y) \mathrm{d} y, \quad \xi_{\star}=\int_{0}^{y_{\star}} g(y) \mathrm{d} y,
$$

and $y_{\star} \neq y_{ \pm}$is the root of $p(y), q(y)$, or $r(y)$ that gives the closest singularity $\xi_{\star} \in \mathbb{C} / \mathbb{Z}$ to the real axis. That is, $y_{\star}$ minimizes $\delta$. The path from $y=0$ to $y=y_{\star}$ in the second integral should be contained in an open simply connected subset of the complex plane where the function $g(y)$ is analytic. See Appendix C for more details about the function $g(y)$ and their domain of analyticity, although that appendix deals with perturbed circles only.

We note that the cusp that appears in the graph of $2 \pi \delta$ for the quartic perturbation correspond to a perturbative parameter $\epsilon$ for which two different roots of $p(y), q(y)$, or $r(y)$ give rise to the same $\delta=\left|\mathfrak{J} \xi_{\star}\right| / C$. 


\section{Perturbed circles}

In this section, we take $b=1$ in the model tables (7). This setting is harder than the one of the perturbed ellipses both in the regular and singular cases. Let us explain it.

We begin with the regular case, so we fix the degree $n \geq 3$ and the period $q \geq 3$ whereas $\epsilon$ tends to zero. First, we note that the Melnikov exponent $c$ in (18) tends to infinity as $b$ tends to one, since $K(0)=\pi / 2$ and $\lim _{m \rightarrow 1^{-}} K(m)=+\infty$. This suggests that the Melnikov method gives little information for perturbed circles. In fact, in [33], it is proved that the first order coefficient $\Delta_{1}^{(1, q)}$ in (16) vanishes for every period $q \notin Q_{n}$, where

$$
Q_{n}= \begin{cases}\{3,5, \ldots, n-2, n\}, & \text { for odd } n, \\ \{2,4, \ldots, n-2, n\} \cup\{2,3, \ldots, n / 2\}, & \text { for even } n\end{cases}
$$

We might use a higher order Melnikov method to look for an order $k=k(n, q) \in \mathbb{N}$ such that

$$
\Delta^{(1, q)}=\epsilon^{k} \Delta_{k}^{(1, q)}+\mathrm{O}\left(\epsilon^{k+1}\right),
$$

with $\Delta_{k}^{(1, q)} \neq 0$. This Melnikov computation is not easy so we have performed a numerical study instead. As before, we do not study $\Delta^{(1, q)}$ but the difference $D_{q}$.

Numerical Result 5. Set

$$
k=k(n, q)= \begin{cases}1+2\left\lceil\frac{q-n}{2 n}\right\rceil, & \text { for odd } n \text { and odd } q, \\ 2\lceil q / 2 n\rceil, & \text { for odd } n \text { and even } q, \\ \lceil 2 q / n\rceil, & \text { for even } n \text { and odd } q, \\ \lceil q / n\rceil, & \text { for even } n \text { and even } q .\end{cases}
$$

If $n \geq 3$ and $q \geq 2$, then there exists $d_{k}=d_{k}(n, q) \neq 0$ such that

$$
D_{q}(\epsilon)=d_{k} \epsilon^{k}+\mathrm{O}\left(\epsilon^{k+1}\right) \text {. }
$$

This numerical result has two nice consequences on the breakup of the resonant caustics of the circular billiard under the perturbation $x^{2}+y^{2}+\epsilon y^{n}=1$ with any fixed degree $n \geq 3$. First, all $(1, q)$-resonant caustics break up, because, once fixed the period $q \geq 2, \Delta^{(1, q)} \neq 0$ for $\epsilon$ small enough. Second, there are breakups of any order, because the map $q \mapsto k(n, q) \in \mathbb{N}$ is exhaustive.

We numerically compute the order $k$ in (23) by noting that

$$
k \simeq \log \left(\frac{D_{q}(\epsilon)}{D_{q}(\epsilon / \mathrm{e})}\right) .
$$

For instance, if $n=7, q=36$, and $\epsilon=10^{-10}$, then we obtain the approximation

$$
k \simeq 5.99999999999999999401 \ldots,
$$

so $k=6$. We have tested the formulas listed in Numerical Result 5 for all degrees $3 \leq n \leq 8$ and all periods $3 \leq q \leq 100$. Note that, once fixed $n$,

$$
k=k(n, q) \asymp \begin{cases}2 q / n, & \text { for even } n \text { and odd } q, \\ q / n, & \text { otherwise, }\end{cases}
$$

as $q \rightarrow+\infty$. Next, we focus on the singular case.
Numerical Result 6. Fix $n \geq 3$. Let $I_{n}$ be the maximal interval defined in (14). If $\epsilon \in I_{n}$, then the Borel transform (4) has a radius of convergence $\rho \in(0,+\infty)$. Set $r=\rho / 2$. There exist two non-zero quasiperiodic functions $A(q)$ and $B(q)$ such that

$$
D_{q} \asymp \begin{cases}B(q) q^{-2} \mathrm{e}^{-2 r q}, & \text { for even } n \text { and odd } q, \\ A(q) q^{-3} \mathrm{e}^{-r q}, & \text { otherwise, }\end{cases}
$$

as $q \rightarrow+\infty$. Besides, there exists $\chi_{n} \in \mathbb{R}$ such that

$$
r=\frac{|\log \epsilon|}{n}+\chi_{n}+\mathrm{o}(1)
$$

as $\epsilon \rightarrow 0$. Finally, there exist a partition $I_{n}=C_{n} \cup P_{n} \cup R_{n}$ satisfying the following properties.

1. $C_{n}$ and $P_{n}$ are open subsets of $I_{n}$, whereas $R_{n}$ is a set of isolated perturbative parameters.

2. If $\epsilon \in C_{n}$, both functions $A(q)$ and $B(q)$ are constant.

3. If $\epsilon \in P_{n}$, both functions $A(q)$ and $B(q)$ are periodic. Namely, they have the form

$$
A(q)=a \cos (2 \pi \beta q), \quad B(q)=\bar{b}+b \cos (4 \pi \beta q),
$$

for some average $\bar{b} \neq 0$, some amplitudes $a, b>0$, and some "shared" frequency $\beta>0$. We note that $\bar{b} \neq b / 2$.

All these numerical results strongly support Conjecture 1. For instance, we conjectured that the function $A(q)$ is either constant: $A(q) \equiv a / 2$, or periodic: $A(q)=a \cos (2 \pi \beta q)$ in open sets of the space of axisymmetric algebraic curves, whereas all other cases are phenomena of co-dimension at least one. This claim agrees with the fact that $C_{n}$ and $P_{n}$ are open subsets of $I_{n}$, whereas $R_{n}$ only contains the perturbative parameters where a transition between constant and periodic cases takes place.

The functions $A(q)$ and $B(q)$ and the exponent $r$ depend on the degree $n$ and the perturbative parameter $\epsilon$, although $B(q)$ is defined only for even $n$. Both functions $A(q)$ and $B(q)$ "share" the frequency in the periodic case. To be precise, the frequency of $B(q)$ is twice the frequency of $A(q)$. It makes sense because the exponent in the asymptotic formula containing the function $B(q)$ is also twice the exponent in the one containing $A(q)$.

The logarithmic behavior of the exponent $r$ stated in (25) is closely related to the asymptotic formula (24). Indeed, if we roughly try to fit the regular behavior (23) when $\epsilon \rightarrow 0$ with the singular behavior $D_{q}=\mathrm{O}\left(q^{m} \mathrm{e}^{-r q}\right)$ when $q \rightarrow+\infty$, then we get

$$
\mathrm{O}\left(q^{m} \mathrm{e}^{-r q}\right)=D_{q}=\mathrm{O}\left(\epsilon^{k}\right) \simeq \mathrm{O}\left(\epsilon^{q / n}\right)=\mathrm{O}\left(\mathrm{e}^{-q|\log \epsilon| / n}\right),
$$

so we guess that $r \simeq|\log \epsilon| / n$. This reasoning is informal but it is confirmed by our experiments. Let us describe them.

We have set $\epsilon \in I_{n} \cap \mathbb{Q}$ in all the experiments. First, we do so because our multiple-precision computations become a bit faster for rational perturbative parameters. There is a second reason for that choice. Namely, we change the precision very often along our computations, and rational values of $\epsilon$ are not affected by such changes, because they are stored as exact numbers. We have also tried to deal with "big" perturbations in order to stress that our results are not perturbative, but we recall 


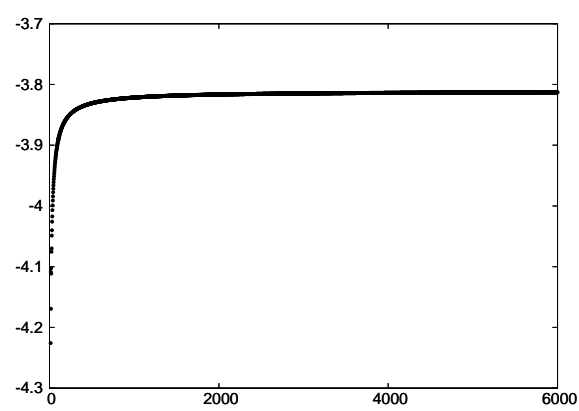

(a) $\hat{D}_{q}$ versus $q$ for $n=4, \epsilon=1$, and odd $q$.

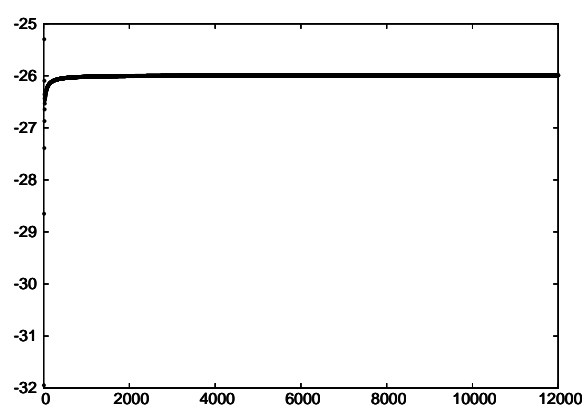

(b) $\hat{D}_{q}$ versus $q$ for $n=4, \epsilon=1$, and even $q$.

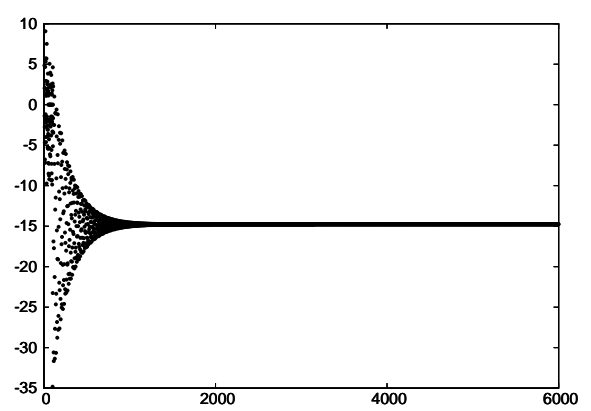

(c) $\hat{D}_{q}$ versus $q$ for $n=7$ and $\epsilon=1 / 1280$.

Figure 8: Examples with a constant asymptotic behavior of the normalized differences $\hat{D}_{q}$.

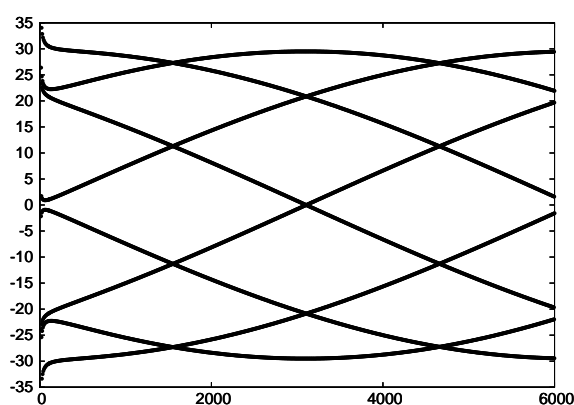

(a) $\hat{D}_{q}$ versus $q$ for $n=3$ and $\epsilon=1 / 3$.

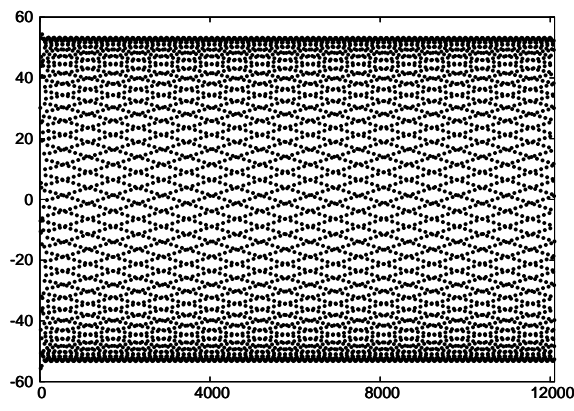

(d) $\hat{D}_{q}$ versus $q$ for $n=6, \epsilon=1$, and even $q$.

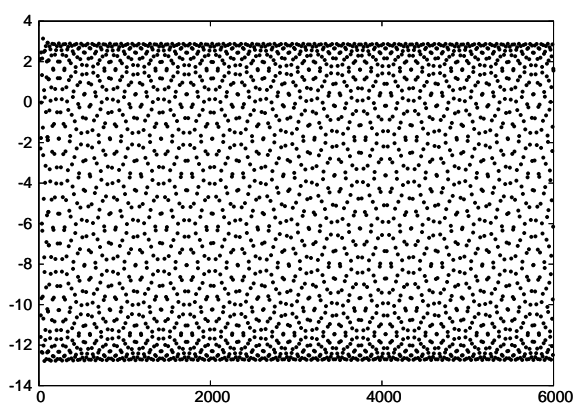

(g) $\hat{D}_{q}$ versus $q$ for $n=6, \epsilon=1$, and odd $q$.

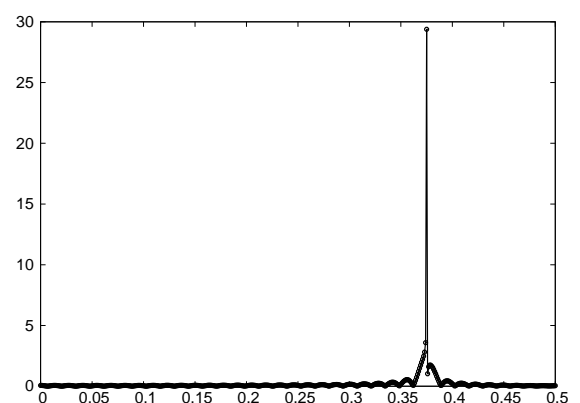

(b) DFT of $\hat{D}_{q}$ for $n=3$ and $\epsilon=1 / 3$.

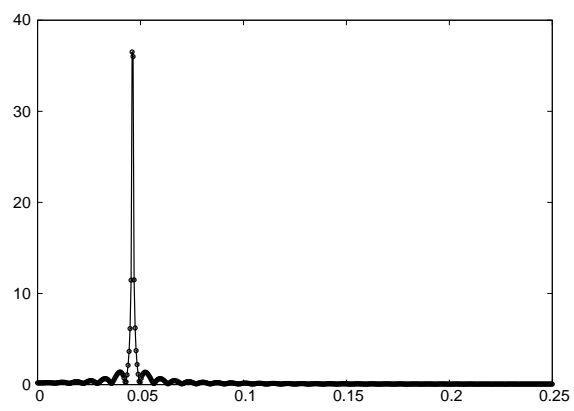

(e) DFT of $\hat{D}_{q}$ for $n=6, \epsilon=1$, and even $q$.

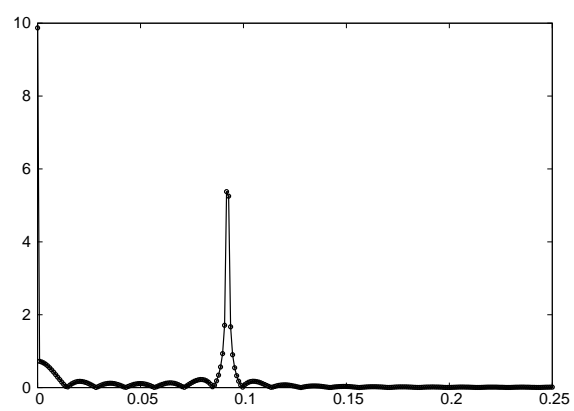

(h) DFT of $\hat{D}_{q}$ for $n=6, \epsilon=1$, and odd $q$.

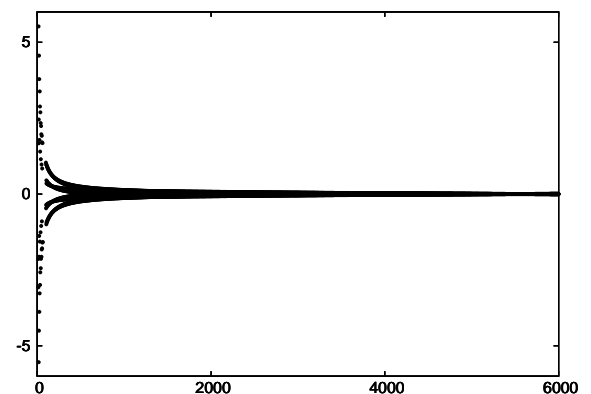

(c) $\hat{D}_{q}-A(q)$ versus $q$ for $n=3$ and $\epsilon=1 / 3$.

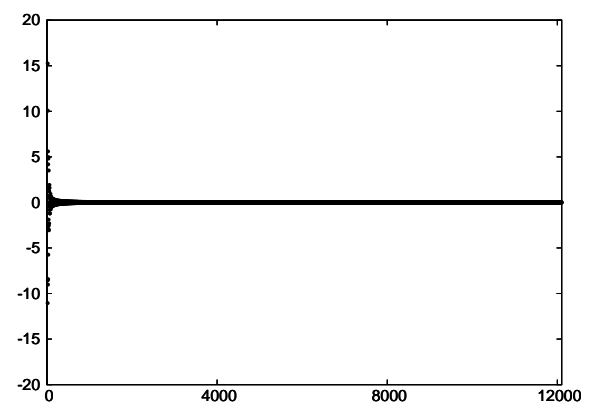

(f) $\hat{D}_{q}-A(q)$ versus $q$ for $n=6, \epsilon=1$, and even $q$.

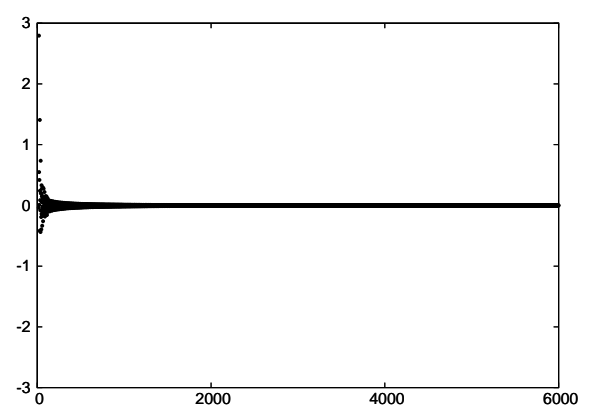

(i) $\hat{D}_{q}-B(q)$ versus $q$ for $n=6, \epsilon=1$, and odd $q$.

Figure 9: Examples with a periodic asymptotic behavior of the normalized differences $\hat{D}_{q}$. We recall that $A(q)=a \cos (2 \pi \beta q)$ and $B(q)=\bar{b}+b \cos (4 \pi \beta q)$. Besides, $a \approx 29.4849$ and $\beta \approx 3 / 8$ in Figure 9(c); $a \approx 53.2369$ and $\beta \approx 0.04614$ in Figure 9(f); and $\bar{b} \approx-4.9257, b \approx 7.80853$, and $\beta \approx 0.04614$ in Figure 9 (i). 
that $\epsilon$ should be smaller than the singular value (15) when $n$ is odd.

First, we compute the exponent $r=\rho / 2$ by using the Borel method, since it is computationally cheaper than the direct one. See Remark 2. Besides, it is not clear how to adapt the direct method when the functions $A(q)$ and $B(q)$ oscillate. We follow the same steps as in the case of perturbed ellipses. However, the Neville extrapolation is more unstable for perturbed circles. In order to overcome this instability, now we take sequences $\left(q_{i}\right)$ of 1000 periods such that $\left|D_{q_{0}}\right| \leq 10^{-5000}$.

Once we find $r$, we compute the normalized differences $\hat{D}_{q}$ already introduced in (22). We have checked that there exist two non-zero quasiperiodic functions $A(q)$ and $B(q)$ such that

$$
\hat{D}_{q} \asymp \begin{cases}B(q), & \text { for even } n \text { and odd } q, \\ A(q), & \text { otherwise, }\end{cases}
$$

as $q \rightarrow+\infty$.

Some paradigmatic examples of the asymptotic behavior of the normalized differences $\hat{D}_{q}$ are displayed in Figures 8 and 9. All these examples are generic in the sense that a small change of the perturbative parameter $\epsilon$ does not produce any qualitative change in the pictures.

For instance, we see three examples where $\hat{D}_{q}$ tends to some constant as $q \rightarrow+\infty$ in Figure 8 . The constant is $A$ in the second and third subfigures, and $B$ in the first one.

We display a first example of periodic asymptotic behavior in Figure 9(a) for the cubic perturbation and $\epsilon=1 / 3$. This value $\epsilon=1 / 3$ is relatively close to the value $\bar{\epsilon}_{3}(1) \approx 0.3849$ where the algebraic curve $x^{2}+y^{2}+\epsilon y^{3}=1$ becomes singular. Next, we compute the discrete Fourier transform (DFT) of the last terms of the sequence $\hat{D}_{q}$. To be precise, the terms in the range $10000<q \leq 12000$ for $n=6$ and even $q$, and in the range $5000<q \leq 6000$ otherwise. We discard the first terms because $\hat{D}_{q} \asymp A(q)$ and $\hat{D}_{q} \asymp B(q)$, so the last normalized differences are closer to the periodic functions we want to determine.

The DFT of the normalized differences $\hat{D}_{q}$ suggests that the periodic function $A(q)$ has a dominant harmonic with amplitude $a \approx 29.4849$ and frequency $\beta \approx 0.375=3 / 8$ when $\epsilon=1 / 3$ and $n=3$. See Figure 9(b). This explains why we see eight waves in Figure 9(a), each one with frequency $|\beta-3 / 8|$. This situation is a source of problems for the following reason. Let us assume that, due to time or computational restrictions, we only compute the normalized differences for periods of the form $q_{i}=q_{0}+8 i$. In that case, we would only see one wave and we would get a wrong frequency. The moral of this story is that we have to compute the normalized differences for all periods. Then we compare the normalized differences $\hat{D}_{q}$ with the cosine wave $A(q)=a \cos (2 \pi \beta q)$ as $q \rightarrow+\infty$. The amplitude $a$ and the frequency $\beta$ are determined by mixing several tools: the DFT, some direct algebraic computations, etcetera. The plot in Figure 9(c) shows that

$$
\lim _{q \rightarrow+\infty}\left(\hat{D}_{q}-A(q)\right)=0
$$

We study the case $n=6$ and $\epsilon=1$ in Figures 9(d)-9(i). The most interesting phenomena shown up by those pictures are the following ones. First, we confirm that the frequency of the periodic function $B(q)$ is twice the frequency of the cosine wave $A(q)$. See Figures 9(e) and 9(h). Second, the average of $B(q)$ is not zero. This is a surprise, because both the periodic functions obtained in similar splitting problems and the periodic function $A(q)$ obtained in this billiard problem have generically zero average. Third, $B(q)=\bar{b}+b \cos (4 \pi \beta q)$, but $\bar{b} \neq b / 2$, which sets another difference with the known asymptotic behaviors for splitting problems.

Next, we present some results about the transition between the two generic — "constant" and "periodic" - asymptotic behaviors of the normalized differences $\hat{D}_{q}$. That is, we intend to visualize what happens at some $\epsilon_{*} \in \partial C_{n} \cap \partial P_{n} \subset R_{n}$.

We focus our attention on the sixtic perturbation: $n=6$. Then the normalized differences have "constant" and "periodic" asymptotic behaviors when $\epsilon \rightarrow 0^{+}$and $\epsilon=1$, respectively. We study the quantities $\hat{D}_{q}$ in a fine grid of perturbative parameters in the interval $(0,1]$. Both functions $A(q)$ and $B(q)$ change at the same transition value. Indeed, we have checked that

$$
(0,472 / 4000] \subset C_{6}, \quad[473 / 4000,1] \subset P_{6},
$$

so the transition takes place at some $\epsilon_{*}=4725 / 40000 \pm 1 / 8000$. We recall that we still do not have a good limit problem to find analytically $\epsilon_{*}$, so we do not compute $\epsilon_{*}$ with more precision. An example of analytical computation of transition values for splitting problems can be found in $[23,26]$.

We display the normalized differences $\hat{D}_{q}$ for

$$
\epsilon=\frac{4600}{40000}, \quad \epsilon=\frac{4700}{40000}, \quad \epsilon=\frac{4725}{40000}, \quad \epsilon=\frac{4750}{40000}
$$

in Figure 10 to see the change of the functions $A(q)$ and $B(q)$. The transition value seems to be very close to the third value. Following Conjecture 1, we guess that, at the transition,

$$
A(q)=a / 2+a \cos (2 \pi \beta q)
$$

for some amplitude $a>0$ and some frequency $\beta>0$, so $A(q)$ oscillates between $-a / 2$ and $3 a / 2$. We see exactly this behavior in Figure 10(e). On the contrary, $B(q)=\bar{b}+b \cos (4 \pi \beta q)$ with $\bar{b} \neq b / 2$ at the transition. See Figure 10(f).

Let us present some numerical results about the logarithmic growth (25) of the exponent $r$. We have computed the exponent $r=\rho / 2$ by using the Borel method for $3 \leq n \leq 8$ in a sequence of perturbative parameters of the form $\epsilon_{j}=2^{-j} / 10$ with $j \geq 0$. We have plotted the results in Figure 11. On the one hand, the curves in Figure 11(a) look like straight lines with slopes $1 / n$, as expected. On the other hand, the curves in Figure 11(b) tend to some constant values $\chi_{n}>0$. This ends the numerical study of such phenomenon.

Finally, we see that our candidate for limit problem captures this logarithmic behavior, although it may not give the exact value of the exponent $r$.

Proposition 7. Let $n \geq 3$ and $\epsilon \in I_{n}$. Let $\kappa(s)$ be the curvature of the strictly convex curve $Q=\left\{(x, y) \in \mathbb{R}^{2}: x^{2}+y^{2}+\epsilon y^{n}=1\right\}$ in some arc-length parameter s. Let $\xi \in \mathbb{R} / \mathbb{Z}$ be the angular 


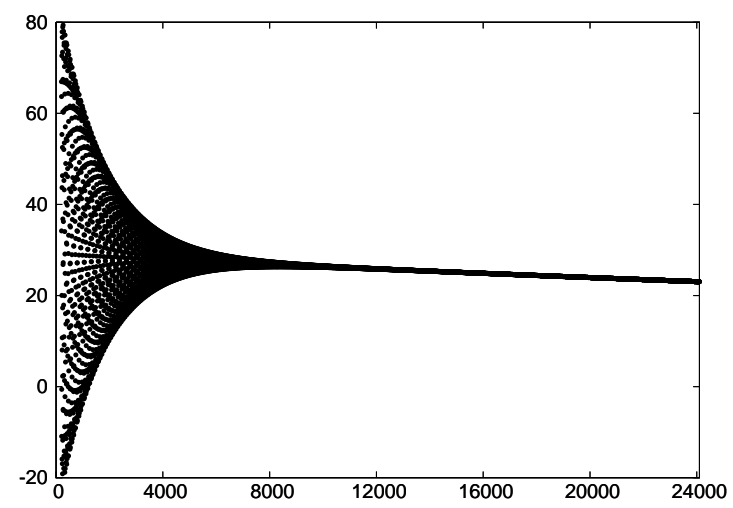

(a) $\epsilon=4600 / 40000$ and even $q$.

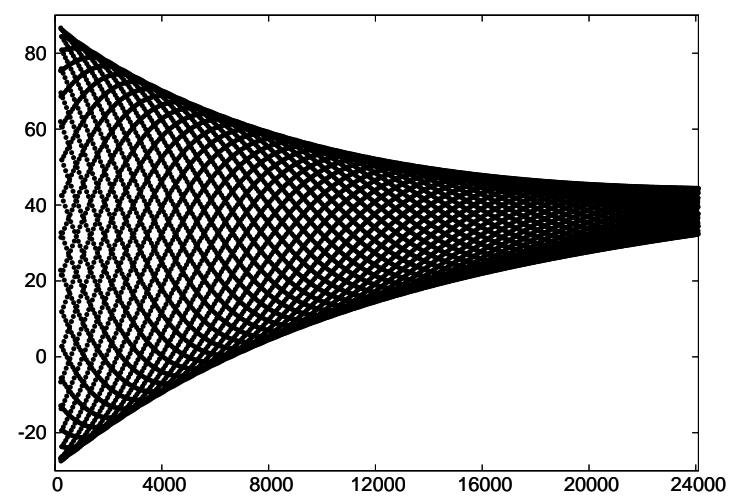

(c) $\epsilon=4700 / 40000$ and even $q$.

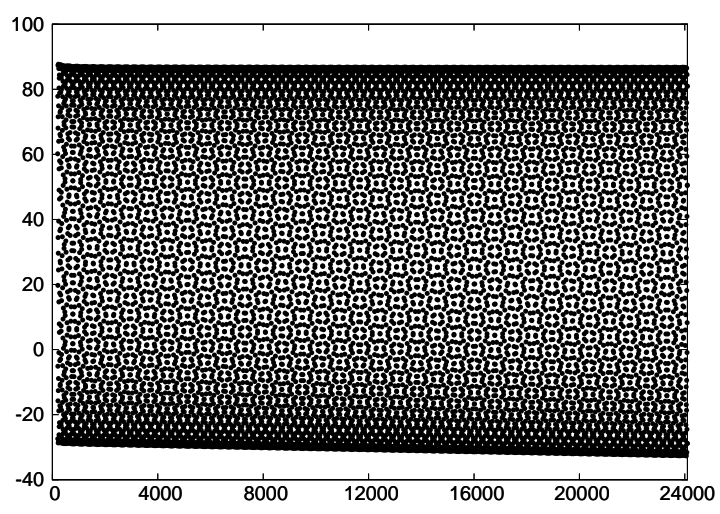

(e) $\epsilon=4725 / 40000$ and even $q$.

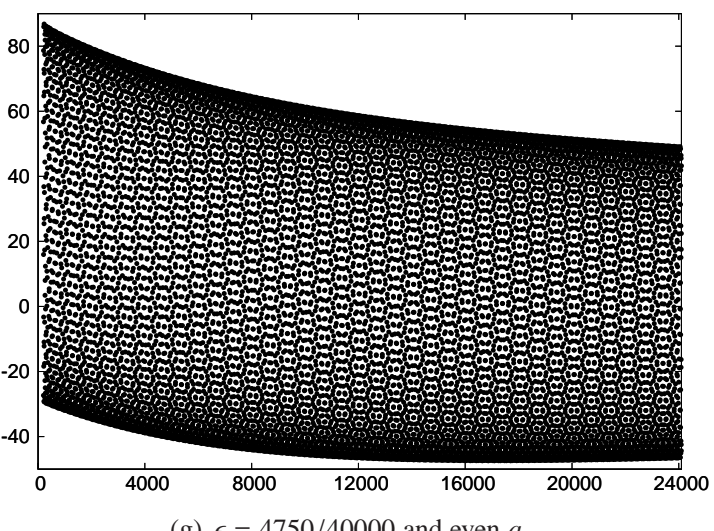

(g) $\epsilon=4750 / 40000$ and even $q$.

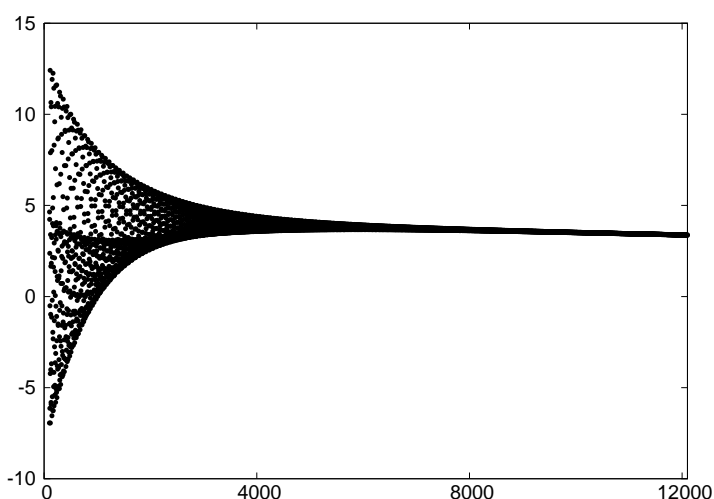

(b) $\epsilon=4600 / 40000$ and odd $q$.

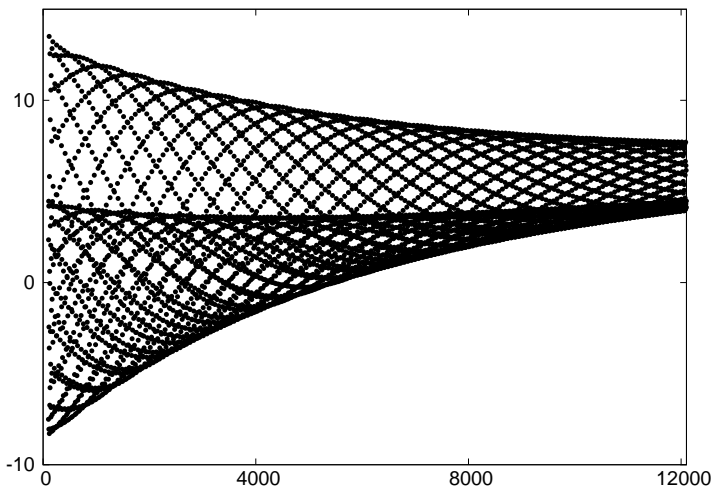

(d) $\epsilon=4700 / 40000$ and odd $q$.

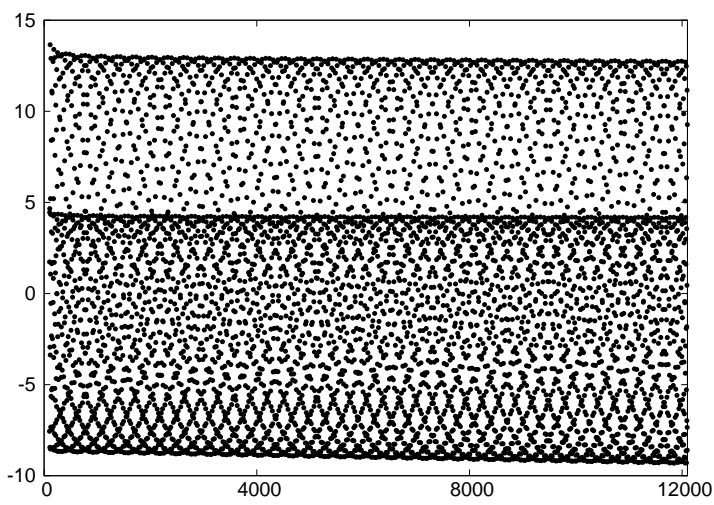

(f) $\epsilon=4725 / 40000$ and odd $q$.

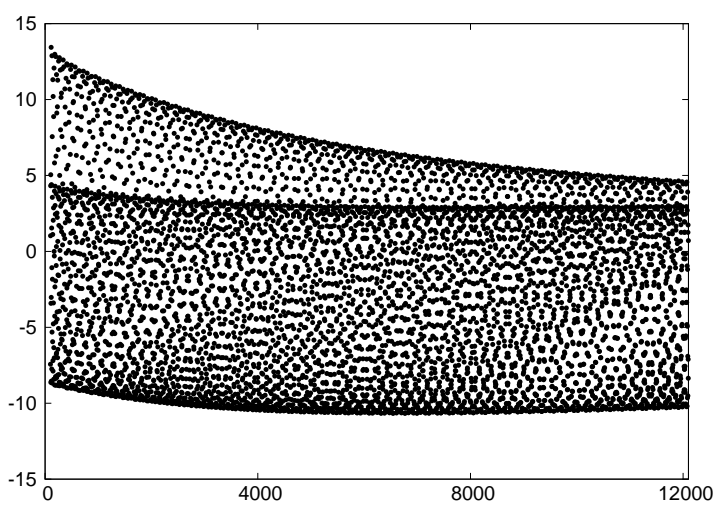

(h) $\epsilon=4750 / 40000$ and odd $q$.

Figure 10: Transition of the functions $A(q)$ (left column) and $B(q)$ (right column) from constant behavior (top row) to periodic behavior (bottom row). The transition takes place approximately at the perturbative parameter used in the third row. We plot the normalized differences $\hat{D}_{q}$ versus $q$ for $n=6$. 


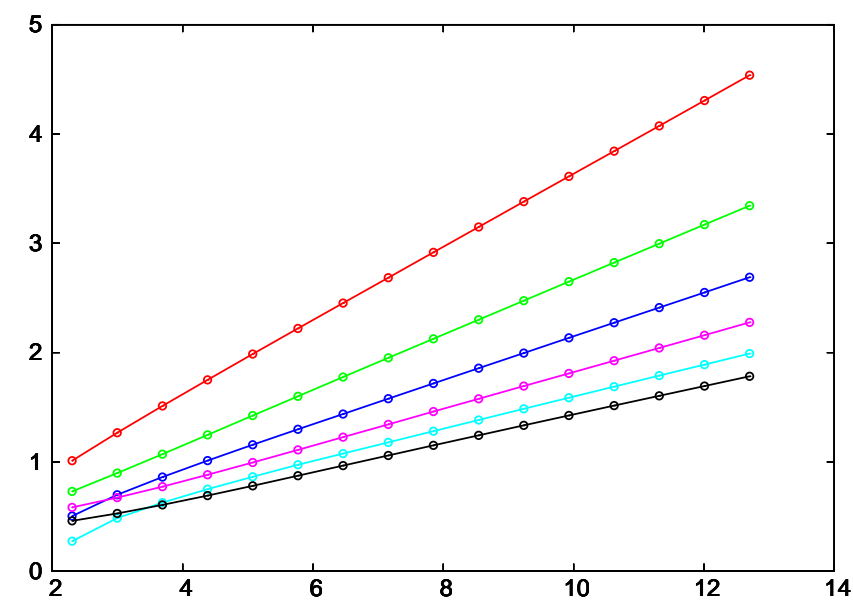

(a) $r$ versus $|\log \epsilon|$.

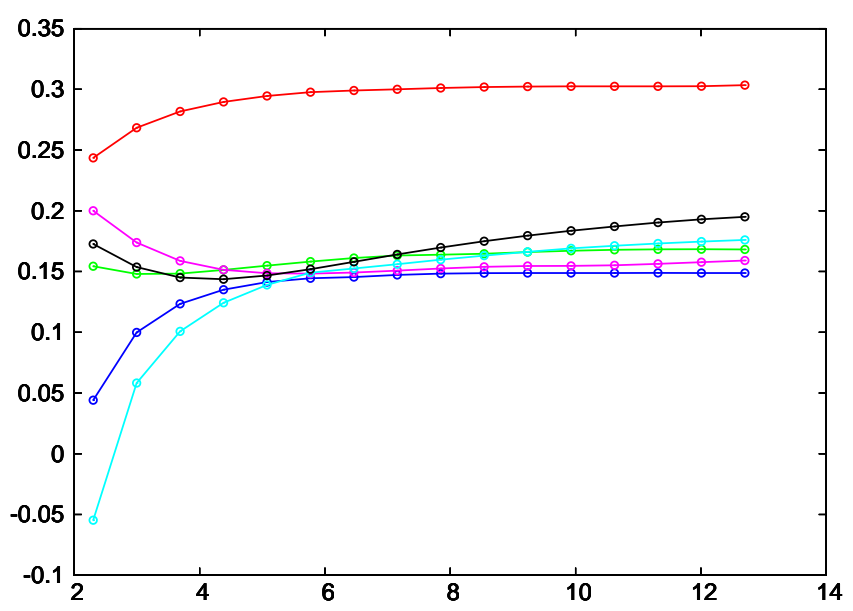

(b) $r-|\log \epsilon| / n$ versus $|\log \epsilon|$.

Figure 11: Logarithmic growth of the exponent $r$ as $\epsilon \rightarrow 0^{+}$. Red: $n=3$. Green: $n=4$. Blue: $n=5$. Magenta: $n=6$. Cyan: $n=7$. Black: $n=8$.

\begin{tabular}{ccc}
\hline \hline$n$ & $\chi_{n}$ & $\eta_{n}$ \\
\hline 3 & $0.30 \ldots$ & $1.1358418243 \ldots$ \\
4 & $0.17 \ldots$ & $1.0703321545 \ldots$ \\
5 & $0.15 \ldots$ & $0.1488295936 \ldots$ \\
6 & $0.15 \ldots$ & $1.0385641059 \ldots$ \\
7 & $0.18 \ldots$ & $0.1823551667 \ldots$ \\
8 & $0.19 \ldots$ & $1.0332248276 \ldots$ \\
\hline \hline
\end{tabular}

Table 3: The constants $\chi_{n}$ and $\eta_{n}$, with $\chi_{n} \leq \eta_{n}$, that appear in formulas (25) and (26), respectively.

variable defined by (9). Let $\delta$ be the distance of the set of singularities and zeros of the curvature $\kappa(\xi)$ to the real axis. There exists $\eta_{n} \in \mathbb{R}$ such that

$$
2 \pi \delta=\frac{|\log \epsilon|}{n}+\eta_{n}+\mathrm{O}\left(\epsilon^{2 / n} \log \epsilon\right)
$$

as $\epsilon \rightarrow 0^{+}$.

The proof of this proposition is placed in Appendix C.

The constant $\chi_{n}$ in (25) is always smaller than (or equal to) the constant $\eta_{n}$ in (26). We compare both constants in Table 3.

Constants $\chi_{n}$ are computed from the numerical data used in Figure 11(b). Constants $\eta_{n}$ are computed by using the techniques explained in Remark 3. On the one hand, we obtain just two significant digits for the constants $\chi_{n}$. On the other hand, we can compute $\eta_{n}$ with a much higher precision; here we have just written their first ten decimal digits. We see that $\chi_{n}<\eta_{n}$ for $n \in\{3,4,6,8\}$. We do not discard the equalities $\chi_{5}=\eta_{5}$ and $\chi_{7}=\eta_{7}$. In order to elucidate them, we compare the exponent $r$ with the quantity $2 \pi \delta$, as we have done before for perturbed ellipses at the end of Section 5. The results are displayed in Figure 12, where we see that our candidate for limit problem gives the exact exponent $r$ in two cases.

To be precise, our numerical results suggest that:

- If $n \in\{3,4,6,8\}$, then $r<2 \pi \delta$ for all $\epsilon \in(0,1 / 10]$; and

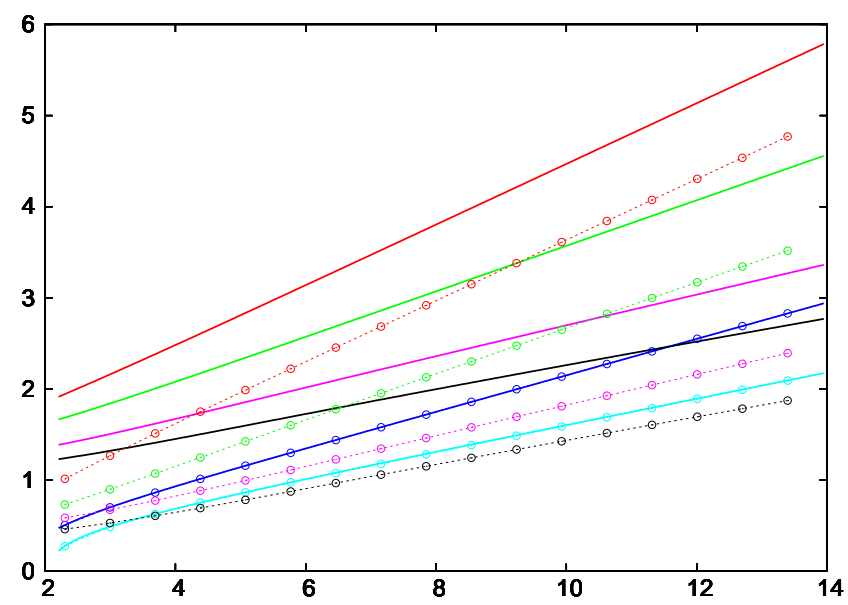

Figure 12: The exponent $r$ (dashed lines with points) and the quantity $2 \pi \delta$ (continuous lines) versus $|\log \epsilon|$. Red: $n=3$. Green: $n=4$. Blue: $n=5$. Magenta: $n=6$. Cyan: $n=7$. Black: $n=8$.

- If $n \in\{5,7\}$, then $r=2 \pi \delta$ for all $\epsilon \in(0,1 / 10]$.

\section{Acknowledgements}

We thank T. M. Seara and C. Simó for very useful remarks and comments. We also appreciate the assistance of A. Granados and P. Roldán in the use of the UPC Applied Math cluster for our experiments.

\section{Appendix A. Proof of Proposition 2}

We will use many properties of elliptic functions listed in the books $[42,43]$, a couple of technical results about elliptic billiards contained in [44, 45], and the subharmonic Melnikov potential of billiards inside perturbed ellipses introduced in [34]. 
We consider the unperturbed ellipse

$$
E=\left\{(x, y) \in \mathbb{R}^{2}: x^{2} / a^{2}+y^{2} / b^{2}=1\right\}, \quad 0<b<a .
$$

It is known that the convex caustics of the billiard inside $E$ are the confocal ellipses

$$
C_{\lambda}=\left\{(x, y) \in \mathbb{R}^{2}: \frac{x^{2}}{a^{2}-\lambda^{2}}+\frac{y^{2}}{b^{2}-\lambda^{2}}=1\right\}, \quad 0<\lambda<b .
$$

There is a unique $(p, q)$-resonant elliptic caustic $C_{\lambda}$ for any relatively prime integers $p$ and $q$ such that $1 \leq p<q / 2$. The caustic parameter of the $(p, q)$-resonant elliptic caustic is implicitly determined by means of equation (A.3).

The complete elliptic integral of the first kind is

$$
K=K(m)=\int_{0}^{\pi / 2}\left(1-m \sin ^{2} \phi\right)^{-1 / 2} \mathrm{~d} \phi .
$$

Its argument $m \in(0,1)$ is called the parameter. We also write $K^{\prime}=K^{\prime}(m)=K(1-m)$. The amplitude function $\varphi=\operatorname{am} t$ is defined through the inversion of the integral

$$
t=\int_{0}^{\varphi}\left(1-m \sin ^{2} \phi\right)^{-1 / 2} \mathrm{~d} \phi
$$

The elliptic sine and elliptic cosine associated to the parameter $m \in(0,1)$ are defined by the trigonometric relations

$$
\operatorname{sn} t=\operatorname{sn}(t, m)=\sin \varphi, \quad \operatorname{cn} t=\operatorname{cn}(t, m)=\cos \varphi .
$$

If the angular variable $\varphi$ changes by $2 \pi$, the angular variable $t$ changes by $4 K$. Thus, any $2 \pi$-periodic function in $\varphi$, becomes $4 K$-periodic in $t$. By abuse of notation, we will also denote the $4 K$-periodic functions with the name of the corresponding $2 \pi$-periodic ones. For example, if $q(\varphi)=(a \cos \varphi, b \sin \varphi)$ is the natural $2 \pi$-periodic parameterization of the ellipse $E$, then $q(t)=(a \mathrm{cn} t, b \operatorname{sn} t)$ is the corresponding $4 K$-periodic parameterization. The billiard dynamics associated to an elliptic caustic $C_{\lambda}$ becomes a rigid rotation $t \mapsto t+\delta$ in the variable $t$. It suffices to find the shift $\delta$ and the parameter $m$ associated to each elliptic caustic $C_{\lambda}$. The parameter $m$ is given in [44, Eq. (3.28)] and the constant shift $\delta$ is given in [44, p. 1543]. We list the formulas in the following lemma.

Lemma 8. Once fixed an elliptic caustic $C_{\lambda}$ with $\lambda \in(0, b)$, the parameter $m \in(0,1)$ and the shift $\delta \in(0,2 K)$ are

$$
m=\frac{a^{2}-b^{2}}{a^{2}-\lambda^{2}}, \quad \delta / 2=\int_{0}^{\vartheta / 2}\left(1-m \sin ^{2} \phi\right)^{-1 / 2} \mathrm{~d} \phi,
$$

where $\vartheta \in(0, \pi)$ is the angle such that $\sin (\vartheta / 2)=\lambda / b$. The segment joining the points $q(t)$ and $q(t+\delta)$ is tangent to $C_{\lambda}$ for all $t \in \mathbb{R}$.

From now on, $m$ and $\delta$ will denote the parameter and the constant shift defined in (A.2). Observe that the elliptic caustic $C_{\lambda}$ is $(p, q)$-resonant if and only if

$$
q \delta=4 K p
$$

This identity has the following geometric interpretation. When a billiard trajectory makes one turn around $C_{\lambda}$, the old angular variable $\varphi$ changes by $2 \pi$, so the new angular variable $t$ changes by $4 K$. Besides, we have seen that the variable $t$ changes by $\delta$ when a billiard trajectory bounces once. Hence, a billiard trajectory inscribed in $E$ and circumscribed around $C_{\lambda}$ makes exactly $p$ turns after $q$ bounces if and only if (A.3) holds.

We consider the elliptic coordinates $(\mu, \varphi)$ associated to the semi-lengths $0<b<a$. That is, $(\mu, \varphi)$ are defined by relations

$$
x=\sigma \cosh \mu \cos \varphi, \quad y=\sigma \sinh \mu \sin \varphi,
$$

where $\sigma=\sqrt{a^{2}-b^{2}}$ is the semi-focal distance of $E$. The ellipse $E$ in these coordinates reads as $\mu \equiv \mu_{0}$, where $\cosh \mu_{0}=a / \sigma$ and $\sinh \mu_{0}=b / \sigma$. Hence, any smooth perturbation of $E$ can be written in elliptic coordinates as

$$
\mu=\mu_{0}+\epsilon \mu_{1}(\varphi)+\mathrm{O}\left(\epsilon^{2}\right)
$$

for some $2 \pi$-periodic function $\mu_{1}: \mathbb{R} \rightarrow \mathbb{R}$.

Lemma 9. Let $p$ and $q$ be two relatively prime integers such that $1 \leq p<q / 2$. Let $C_{\lambda}$ be the $(p, q)$-resonant elliptic caustic of the ellipse (A.1). Let

$$
\Delta^{(p, q)}=\epsilon \Delta_{1}^{(p, q)}+\mathrm{O}(\epsilon)
$$

be the maximal difference among lengths of $(p, q)$-periodic trajectories inside the perturbed ellipse (A.5). Let $\mu_{1}(t)$ be the $4 K$ periodic function associated to the $2 \pi$-periodic one $\mu_{1}(\varphi)$. Let

$$
L_{1}^{(p, q)}(t)=2 \lambda \sum_{j=0}^{q-1} \mu_{1}(t+j \delta)
$$

be the subharmonic Melnikov potential of the caustic $C_{\lambda}$ for the perturbed ellipse (A.5). If $L_{1}^{(p, q)}(t)$ does not have degenerate critical points and $\epsilon>0$ is small enough, then there is a one-toone correspondence between the critical points of $L_{1}^{(p, q)}(t)$ and the ( $p, q)$-periodic billiard trajectories inside (A.5). Besides,

$$
\Delta_{1}^{(p, q)}=\max L_{1}^{(p, q)}-\min L_{1}^{(p, q)} .
$$

Proof. It follows directly from results contained in [34].

We will determine the asymptotic behavior of $\Delta_{1}^{(p, q)}$. First, we study the asymptotic behavior of the $(p, q)$-resonant caustic $C_{\lambda}$ as $p / q \rightarrow 0^{+}$.

Lemma 10. If $C_{\lambda}$ is the $(p, q)$-resonant elliptic caustic of the ellipse (A.1), then $\lambda \asymp \Xi p / q$ as $p / q \rightarrow 0^{+}$, where

$$
\Xi=\Xi(a, b):=a b \int_{b^{2}}^{a^{2}}\left(s\left(s-b^{2}\right)\left(a^{2}-s\right)\right)^{-1 / 2} \mathrm{~d} s .
$$

Proof. It follows directly from [45, Proposition 10].

Lemma 11. The following properties hold for $\mu_{1}(\varphi)=\cos ^{2} \varphi$.

1. The Melnikov potential $L_{1}^{(p, q)}(t)$ has just two real critical points (modulo its periodicity), none of them degenerate. 
2. There exist an exponent $\zeta=\zeta\left(\omega_{*}, a, b\right)>0$ and a quantity $\Omega_{4}=\Omega_{4}\left(\omega_{*}, a, b, p, q\right)>0$ such that

$$
\Delta_{1}^{(p, q)} \asymp \begin{cases}2 \Omega_{4} \mathrm{e}^{-2 \zeta q}, & \text { for odd } q, \\ \Omega_{4} \mathrm{e}^{-\zeta q}, & \text { for even } q,\end{cases}
$$

as $p / q \rightarrow \omega_{*} \in\{0\} \cup((0,1) \backslash \mathbb{Q})$.

3. There exist $\Gamma_{4}=\Gamma_{4}\left(\omega_{*}, a, b\right)>0$ and $\Theta_{4}=\Theta_{4}(a, b)>0$ such that

$$
\Omega_{4}\left(\omega_{*}, a, b, p, q\right)= \begin{cases}\Gamma_{4} q^{2}, & \text { if } \omega_{*} \in(0,1) \backslash \mathbb{Q}, \\ \Theta_{4} p q, & \text { if } \omega_{*}=0 .\end{cases}
$$

4. $\zeta(0, a, b)=\pi K^{\prime}\left(1-(b / a)^{2}\right) / 2 K\left(1-(b / a)^{2}\right)$.

Proof. By definition, if $\mu_{1}(\varphi)=\cos ^{2} \varphi$, then

$$
L_{1}^{(p, q)}(t)=2 \lambda \sum_{j=0}^{q-1} \mathrm{cn}^{2}(t+j \delta)
$$

The square of the elliptic cosine is an elliptic function of order two, periods $2 K$ and $2 K^{\prime} i$, and double poles in the set

$$
P=K^{\prime} \mathrm{i}+2 K \mathbb{Z}+2 K^{\prime} \mathrm{i} \mathbb{Z}
$$

Besides, the principal part of any pole $\tau \in P$ is $-m^{-1}(t-\tau)^{-2}$. In particular, $L_{1}^{(p, q)}(t)$ is also an elliptic function of order two, and so, it can be determined (modulo an additive constant) by its periods, poles, and principal parts.

We study the cases odd $q$ and even $q$ separately.

If $q$ is odd, then $L_{1}^{(p, q)}(t)$ has periods $2 K / q$ and $2 K^{\prime} \mathrm{i}$ and double poles with principal parts $-2 \lambda m^{-1}(t-\tau)^{-2}$ in the set

$$
P_{q}=K^{\prime} \mathrm{i}+\frac{2 K}{q} \mathbb{Z}+2 K^{\prime} \mathrm{i} .
$$

It is known that $K^{\prime}(m) / K(m)$ is a decreasing function such that

$$
\lim _{m \rightarrow 0^{+}} \frac{K^{\prime}(m)}{K(m)}=+\infty, \quad \lim _{m \rightarrow 1^{-}} \frac{K^{\prime}(m)}{K(m)}=0 .
$$

Therefore, there exists a unique $m_{q} \in(0,1)$ such that

$$
\frac{K_{q}^{\prime}}{K_{q}}:=\frac{K^{\prime}\left(m_{q}\right)}{K\left(m_{q}\right)}=q \frac{K^{\prime}(m)}{K(m)}=: q \frac{K^{\prime}}{K} .
$$

Henceforth, we write that $K=K(m), K^{\prime}=K^{\prime}(m), K_{q}=K\left(m_{q}\right)$, and $K_{q}^{\prime}=K^{\prime}\left(m_{q}\right)$ for short. Thus,

$$
L_{1}^{(p, q)}(t)=\text { const. }+2 \lambda\left(q K_{q} / K\right)^{2}\left(m_{q} / m\right) \mathrm{cn}^{2}\left(q K_{q} t / K, m_{q}\right),
$$

which has just two real critical points (modulo its periodicity), none of them degenerate. Besides

$$
\Delta_{1}^{(p, q)}=\max L_{1}^{(p, q)}-\min L_{1}^{(p, q)}=2 \lambda\left(q K_{q} / K\right)^{2}\left(m_{q} / m\right) .
$$

If $p / q \rightarrow \omega_{*} \in(0,1) \backslash \mathbb{Q}$, then $q \rightarrow+\infty$ and $\lambda \rightarrow \lambda_{*} \in(0, b)$, where $C_{\lambda_{*}}$ is the elliptic caustic with rotation number $\omega_{*}$, so

$$
\begin{aligned}
m & \rightarrow m_{*}:=\frac{a^{2}-b^{2}}{a^{2}-\lambda_{*}^{2}} \in(0,1), \quad m_{q} \rightarrow 0^{+}, \\
\frac{K^{\prime}}{K} \rightarrow \frac{K_{*}^{\prime}}{K_{*}}:=\frac{K^{\prime}\left(m_{*}\right)}{K\left(m_{*}\right)} \in(0,+\infty), & K_{q} \rightarrow \frac{\pi}{2} .
\end{aligned}
$$

Using [42, 17.3.14 \& 17.3.16], we get the asymptotic formula $m_{q} \asymp 16 \mathrm{e}^{-2 \zeta q}$, where $\zeta:=\pi K_{*}^{\prime} / 2 K_{*}$. Finally, we obtain that

$$
\Delta_{1}^{(p, q)} \asymp \frac{8 \pi^{2} \lambda_{*}}{m_{*} K_{*}^{2}} q^{2} \mathrm{e}^{-2 \zeta q} \text {, as } p / q \rightarrow \omega_{*} \text { and } q \text { is odd. }
$$

If $q$ is even, then $\mathrm{cn}^{2}(t+q \delta / 2, m)=\mathrm{cn}^{2}(t, m)$ and

$$
L_{1}^{(p, q)}(t)=4 \lambda \sum_{j=0}^{q / 2-1} \mathrm{cn}^{2}(t+j \delta, m)
$$

so $L_{1}^{(p, q)}(t)$ has periods $4 K / q$ and $2 K^{\prime} \mathrm{i}$. In this case,

$$
\Delta_{1}^{(p, q)} \asymp \frac{4 \pi^{2} \lambda_{*}}{m_{*} K_{*}^{2}} q^{2} \mathrm{e}^{-\zeta q}, \text { as } p / q \rightarrow \omega_{*} \text { and } q \text { is even. }
$$

Next, we study the case $\omega_{*}=0$, when the $(p, q)$-periodic orbits approach the boundary. In this case,

$$
\lambda_{*}=0, \quad m_{*}=1-(b / a)^{2}, \quad \zeta(0, a, b)=\frac{\pi K^{\prime}\left(1-(b / a)^{2}\right)}{2 K\left(1-(b / a)^{2}\right)} .
$$

Since $\lambda_{*}=0$, we need the asymptotic behavior of the caustic parameter $\lambda$ as $p / q \rightarrow 0^{+}$. We recall that $\lambda \asymp \Xi p / q$ in that case, where $\Xi=\Xi(a, b)$ is the integral defined in (A.6). Hence,

$$
\Gamma_{4}=\frac{4 \pi^{2} \lambda_{*}}{m_{*} K_{*}^{2}}, \quad \Theta_{4}=\frac{4 \pi^{2} \Xi(a, b)}{\left(1-(b / a)^{2}\right) K\left(1-(b / a)^{2}\right)^{2}},
$$

and this ends the proof of the lemma.

Lemma 12. The following properties hold for $\mu_{1}(\varphi)=-\sin \varphi$.

1. If $q$ is even, then $L_{1}^{(p, q)}(t) \equiv 0$ and $\Delta_{1}^{(p, q)}=0$.

2. If $q$ is odd, then $L_{1}^{(p, q)}(t)$ has just two real critical points (modulo its periodicity), none of them degenerate.

3. Let $\zeta\left(\omega_{*}, a, b\right)$ be the exponent introduced in Lemma 11. If $q$ is even, then there exists $\Omega_{3}=\Omega_{3}\left(\omega_{*}, a, b, p, q\right)>0$ such that

$$
\Delta_{1}^{(p, q)} \asymp \Omega_{3} \mathrm{e}^{-\zeta q}, \quad p / q \rightarrow \omega_{*} \in\{0\} \cup((0,1) \backslash \mathbb{Q}) .
$$

4. There exist $\Gamma_{3}=\Gamma_{3}\left(\omega_{*}, a, b\right)>0$ and $\Theta_{3}=\Theta_{3}(a, b)>0$ such that

$$
\Omega_{3}\left(\omega_{*}, b, a, p, q\right)= \begin{cases}\Gamma_{3} q, & \text { if } \omega_{*} \in(0,1) \backslash \mathbb{Q}, \\ \Theta_{3} p, & \text { if } \omega_{*}=0\end{cases}
$$

Proof. If $q$ is even, then $p$ is odd, $\operatorname{sn}(t+\delta / 2)=-\operatorname{sn} t$, and $L_{1}^{(p, q)}(t)=-2 \lambda \sum_{j=0}^{q-1} \operatorname{sn}(t+j \delta) \equiv 0$.

The case odd $q$ follows the lines of the proof of Lemma 11. The constants are

$$
\Gamma_{3}=\frac{8 \pi \lambda_{*}}{\sqrt{m_{*}} K_{*}}, \quad \Theta_{3}=\frac{8 \pi \Xi(a, b)}{\left(1-(b / a)^{2}\right)^{1 / 2} K\left(1-(b / a)^{2}\right)},
$$

where $C_{\lambda_{*}}$ is the elliptic caustic with rotation number $\omega_{*}, m_{*}=$ $\left(a^{2}-b^{2}\right) /\left(a^{2}-\lambda_{*}^{2}\right)$, and $K_{*}=K\left(m_{*}\right)$. We omit the details.

Next, we relate the original perturbed ellipses (7) written in Cartesian coordinates, to the perturbed ellipses (A.5) written in elliptic coordinates (A.4). 
Lemma 13. Set $0<b<a$.

1. The perturbed ellipse (A.5) with $\mu_{1}(\varphi)=-\sin \varphi$ has, up to terms of second order in $\epsilon$, the implicit equation

$$
\frac{x^{2}}{a^{2}}+\frac{\left(y-\epsilon b^{2} / a\right)^{2}}{b^{2}}+2 \frac{a^{2}-b^{2}}{b^{4}} \epsilon y^{3}=1 .
$$

2. The perturbed ellipse (A.5) with $\mu_{1}(\varphi)=\cos ^{2} \varphi$ has, up to terms of second order in $\epsilon$, the implicit equation

$$
\frac{x^{2}}{\alpha^{2}}+\frac{y^{2}}{\beta^{2}}+2 \frac{a^{2}-b^{2}}{b^{5}} \epsilon y^{4}=1,
$$

for some semi-lengths $\alpha=a+\mathrm{O}(\epsilon)$ and $\beta=b+\mathrm{O}(\epsilon)$.

Proof. Let $P_{1}: \mathbb{R}^{2} \rightarrow \mathbb{R}$ be a smooth function. The perturbed ellipse written in Cartesian coordinates as

$$
x^{2} / a^{2}+y^{2} / b^{2}+\epsilon P_{1}(x, y)+\mathrm{O}\left(\epsilon^{2}\right)=1,
$$

and the perturbed ellipse written in elliptic coordinates as (A.5) are linked through relation

$$
2\left(a^{2} \sin ^{2} \varphi+b^{2} \cos ^{2} \varphi\right) \mu_{1}(\varphi)+a b P_{1}(a \cos \varphi, b \sin \varphi)=0 .
$$

The rest of the proof is a tedious, but straightforward, computation.

Finally, we get the claims stated in Proposition 2 from the previous results by using that $\alpha=a+\mathrm{O}(\epsilon)$ and $\beta=b+\mathrm{O}(\epsilon)$ and by taking $a=1$. To be precise, then

$$
\begin{aligned}
c & =c(b)=\zeta(0,1, b)=\frac{\pi K^{\prime}\left(1-b^{2}\right)}{2 K\left(1-b^{2}\right)}, \\
M_{3} & =M_{3}(b)=\frac{b^{4} \Theta_{3}(1, b)}{2\left(1-b^{2}\right)}=\frac{4 \pi b^{4} \Xi(1, b)}{\left(1-b^{2}\right)^{3 / 2} K\left(1-b^{2}\right)}, \\
M_{4} & =M_{4}(b)=\frac{b^{5} \Theta_{4}(1, b)}{2\left(1-b^{2}\right)}=\frac{2 \pi^{2} b^{5} \Xi(1, b)}{\left(1-b^{2}\right)^{2} K\left(1-b^{2}\right)^{2}},
\end{aligned}
$$

where the elliptic integral $\Xi=\Xi(a, b)$ is defined in (A.6).

\section{Appendix B. Proof of Proposition 4}

We parameterize the ellipse by using the angular variable $\varphi$. That is, we use the parametrization $\sigma(\varphi)=(\cos \varphi, b \sin \varphi)$. The curvature of the ellipse $E$ at the point $\sigma(\varphi)$ is

$$
\kappa(\varphi)=\frac{b}{\left(\sin ^{2} \varphi+b^{2} \cos ^{2} \varphi\right)^{3 / 2}}=\frac{1}{b^{2}\left(1+v \sin ^{2} \varphi\right)^{3 / 2}},
$$

where $v=\left(1-b^{2}\right) / b^{2}>0$. The arc-length parameter $s$ and the angular parameter $\varphi$ are related by

$$
\frac{\mathrm{d} s}{\mathrm{~d} \varphi}(\varphi)=\left\|\sigma^{\prime}(\varphi)\right\|=\sqrt{\sin ^{2} \varphi+b^{2} \cos ^{2} \varphi}=b \sqrt{1+v \sin ^{2} \varphi}
$$

First, we compute the constant

$$
\begin{aligned}
C & =\int_{E} \kappa^{2 / 3} \mathrm{~d} s=4 b^{-1 / 3} \int_{0}^{\pi / 2}\left(1+v \sin ^{2} \varphi\right)^{-1 / 2} \mathrm{~d} \varphi \\
& =4 b^{-1 / 3} K(-v)=4 b^{2 / 3} K\left(1-b^{2}\right) .
\end{aligned}
$$

We have used [42, 17.4.17] in the last equality.

The incomplete elliptic integral of the first kind with amplitude $\varphi \in(0, \pi / 2)$ and parameter $m \in(0,1)$ is

$$
F(\varphi \mid m)=\int_{0}^{\varphi}\left(1-m \sin ^{2} \theta\right)^{-1 / 2} \mathrm{~d} \theta .
$$

This definition can be extended to complex amplitudes and any real parameter [42]. Note that $F(\pi / 2 \mid m)=K(m)$.

The curvature $\kappa(\varphi)$ has no complex zeros but has complex singularities at the points such that $\sin ^{2} \varphi=-1 / v$. This equation becomes $\sinh ^{2} \psi=1 / v$ under the change $\varphi=\mathrm{i} \psi$. Let $\psi_{*}$ be the only positive solution of the previous equation. Any singularity of $\kappa(\varphi)$ has the form

$$
\varphi=\varphi_{n}^{ \pm}:= \pm \mathrm{i} \psi_{*}+n \pi, \quad n \in \mathbb{Z} .
$$

Let $\xi_{n}^{ \pm}$be the complex singularity of $\kappa(\xi)$ associated to $\varphi_{n}^{ \pm}$ through the change of variables

$$
\xi=C^{-1} \int_{0}^{s} \kappa^{2 / 3}(t) \mathrm{d} t=C^{-1} \int_{0}^{\varphi} \kappa^{2 / 3}(\theta) \frac{\mathrm{d} s}{\mathrm{~d} \varphi}(\theta) \mathrm{d} \theta .
$$

The complex path in this integral is the segment from 0 to $\varphi$.

Next, we compute the complex singularities $\xi_{n}^{+}$:

$$
\begin{aligned}
\xi_{n}^{+} & =C^{-1} \int_{0}^{\varphi_{n}^{+}} \kappa^{2 / 3}(\theta) \frac{\mathrm{d} s}{\mathrm{~d} \varphi}(\theta) \mathrm{d} \theta \\
& =C^{-1} b^{-1 / 3} F\left(\mathrm{i} \psi_{*}+n \pi \mid-v\right) \\
& =2 n C^{-1} b^{-1 / 3} K(-v)+\mathrm{i} C^{-1} b^{-1 / 3} F\left(\pi / 2 \mid b^{2}\right) \\
& =2 n C^{-1} b^{2 / 3} K\left(1-b^{2}\right)+\mathrm{i} C^{-1} b^{2 / 3} K\left(b^{2}\right) \\
& =n / 2+\mathrm{i} C^{-1} b^{2 / 3} K^{\prime}\left(1-b^{2}\right) .
\end{aligned}
$$

By symmetry, $\xi_{n}^{-}=-\xi_{-n}^{+}$. We have used formula [42, 17.4.3] to compute $F\left(\mathrm{i} \psi_{*}+n \pi \mid-v\right)$, formula $[42,17.4 .8]$ to compute $F(\mathrm{i} \psi * \mid-v)$, and formula $[42,17.4 .15]$ to compute $F\left(\pi / 2 \mid b^{2}\right)$.

Therefore, the distance $\delta$ of the set of singularities and zeros of the curvature $\kappa(\xi)$ to the real axis is

$$
\delta=C^{-1} b^{2 / 3} K^{\prime}\left(1-b^{2}\right)=\frac{K^{\prime}\left(1-b^{2}\right)}{4 K\left(1-b^{2}\right)}=c / 2 \pi .
$$

\section{Appendix C. Proof of Proposition 7}

Fix the integer $n \geq 3$. We consider the perturbed circles

$$
Q=\left\{(x, y) \in \mathbb{R}^{2}: x^{2}+y^{2}+\epsilon y^{n}=1\right\}
$$

where $0<\epsilon \ll 1$ is a small perturbative parameter.

Let $C=C(\epsilon)$ be the constant defined in (9). If $\epsilon=0$, then $Q$ is a circle of radius one with curvature $\kappa \equiv 1$, so

$$
C(0)=\int_{Q} \kappa^{2 / 3} \mathrm{~d} s=\int_{Q} \mathrm{~d} s=\operatorname{Length}(Q)=2 \pi .
$$

We note that (C.1) is a smooth perturbation of a circle of radius one, so $C(\epsilon)$ is smooth at $\epsilon=0$ and

$$
C=C(\epsilon)=C(0)+\mathrm{O}(\epsilon)=2 \pi+\mathrm{O}(\epsilon) .
$$


We introduce the polynomial $r(y)=1-y^{2}-\epsilon y^{n}$. Note that $(x, y) \in Q$ if and only if $x^{2}=r(y)$. By taking derivatives twice with respect to $y$ the implicit relation $x^{2}=r(y)$, we get the auxiliary polynomials

$$
\begin{aligned}
p(y) & =-x^{3} \frac{\mathrm{d}^{2} x}{\mathrm{~d} y^{2}}=\left(\frac{r^{\prime}(y)}{2}\right)^{2}-\frac{r(y) r^{\prime \prime}(y)}{2} \\
& =1+\epsilon p_{n-2} y^{n-2}+\epsilon p_{n} y^{n}+\epsilon^{2} p_{2 n-2} y^{2 n-2}, \\
q(y) & =x^{2}+\left(x \frac{\mathrm{d} x}{\mathrm{~d} y}\right)^{2}=r(y)+\left(\frac{r^{\prime}(y)}{2}\right)^{2} \\
& =1+\epsilon q_{n} y^{n}+\epsilon^{2} q_{2 n-2} y^{2 n-2},
\end{aligned}
$$

whose coefficients are $p_{n-2}=n(n-1) / 2, p_{n}=-(n-1)(n-2) / 2$, $p_{2 n-2}=-n(n-2) / 4, q_{n}=n-1$, and $q_{2 n-2}=n^{2} / 4$. The length element and the curvature at the point $(x, y) \in Q$ are

$$
\begin{aligned}
\mathrm{d} s & =\sqrt{1+\left(\frac{\mathrm{d} x}{\mathrm{~d} y}\right)^{2}} \mathrm{~d} y=\sqrt{\frac{q(y)}{r(y)}} \mathrm{d} y, \\
\kappa & =-\frac{\mathrm{d}^{2} x}{\mathrm{~d} y^{2}}\left(1+\left(\frac{\mathrm{d} x}{\mathrm{~d} y}\right)^{2}\right)^{-3 / 2}=\frac{p(y)}{q^{3 / 2}(y)} .
\end{aligned}
$$

The curvature should be positive, which explains the minus sign in the formula for $k(y)$. Thus, we can relate any singularity (or any zero) $y_{\star} \in \mathbb{C}$ of the curvature $\kappa(y)$, with the corresponding singularities (or zeros) $s_{\star} \in \mathbb{C} / l \mathbb{Z}$ and $\xi_{\star} \in \mathbb{C} / \mathbb{Z}$ by means of the formula

$$
\xi_{\star}=\int_{0}^{s_{\star}} \kappa^{2 / 3}(s) \mathrm{d} s=\int_{0}^{y_{\star}} g(y) \mathrm{d} y
$$

where

$$
g(y):=\kappa^{2 / 3}(y) \frac{\mathrm{d} s}{\mathrm{~d} y}(y)=\frac{p^{2 / 3}(y)}{\sqrt{r(y) q(y)}} .
$$

Let $\mathcal{R} \subset \mathbb{C}$ be the union of the complex rays $\left\{\alpha y_{0}: \alpha \geq 0\right\}$, where $y_{0}$ is a root of $p(y), q(y)$ or $r(y)$. The function $g(y)$ is analytic in $\mathbb{C} \backslash \mathcal{R}$, so we will avoid the set $\mathcal{R}$ when computing the integral $\int_{0}^{y_{\star}} g(y) \mathrm{d} y$ along complex paths.

Lemma 14. Let $0<\epsilon \ll 1$ and $n \in \mathbb{N}$ with $n \geq 3$.

1. The polynomial $p(y)$ has $n$ roots of the form

$$
z \epsilon^{-1 / n}+\mathrm{O}\left(\epsilon^{1 / n}\right), \quad z^{n}=2 /((n-1)(n-2)) ;
$$

and $n-2$ roots of the form

$$
z \epsilon^{-1 /(n-2)}+\mathrm{O}\left(\epsilon^{1 /(n-2)}\right), \quad z^{n-2}=-(n-1)(n-2) / n .
$$

2. The polynomial $q(y)$ has $n$ roots of the form

$$
z \epsilon^{-1 / n}+\mathrm{O}\left(\epsilon^{1 / n}\right), \quad z^{n}=-1 /(n-1) ;
$$

and $n-2$ roots of the form

$$
z \epsilon^{-1 /(n-2)}+\mathrm{O}\left(\epsilon^{1 /(n-2)}\right), \quad z^{n-2}=-4(n-1) / n^{2} .
$$

3. The polynomial $r(y)$ has $n-2$ roots of the form

$$
z \epsilon^{-1 /(n-2)}+\mathrm{O}\left(\epsilon^{1 /(n-2)}\right), \quad z^{n-2}=-1
$$

and two real roots of the form $y_{ \pm}= \pm 1+\mathrm{O}(\epsilon)$.
Besides, each one of these roots depends on some positive fractional power of $\epsilon$ in an analytic way.

Proof. If $w_{0}(z)$ is a polynomial with a simple root $z_{0}$ and $w_{1}(z)$ is another polynomial, then $w(z)=w_{0}(z)+\mu w_{1}(z)$ has some root of the form $z=z_{0}+\mathrm{O}(\mu)$ which depends analytically on $\mu$. The roots $y_{ \pm}= \pm 1+\mathrm{O}(\epsilon)$ of the polynomial $r(y)=1-y^{2}-\epsilon y^{n}$ are obtained directly with $w_{0}(z)=1-z^{2}, w_{1}(z)=-z^{n}$, and $\mu=\epsilon$.

If we take $\mu=\epsilon^{2 / n}$, then

$$
\begin{aligned}
& p\left(\epsilon^{-1 / n} z\right)=1+p_{n} z^{n}+\mu\left(p_{n-2} z^{n-2}+p_{2 n-2} z^{2 n-2}\right), \\
& q\left(\epsilon^{-1 / n} z\right)=1+q_{n} z^{n}+\mu q_{2 n-2} z^{2 n-2},
\end{aligned}
$$

and we find the $n$ roots with an $\mathrm{O}\left(\epsilon^{-1 / n}\right)$-modulus of $p(y)$ and the $n$ roots with an $\mathrm{O}\left(\epsilon^{-1 / n}\right)$-modulus of $q(y)$.

If we take $\mu=\epsilon^{2 /(n-2)}$, then

$$
\begin{aligned}
& \mu p\left(\epsilon^{-1 /(n-2)} z\right)=z^{n}\left(p_{n}+p_{2 n-2} z^{n-2}\right)+\mu\left(1+p_{n-2} z^{n-2}\right), \\
& \mu q\left(\epsilon^{-1 /(n-2)} z\right)=z^{n}\left(q_{n}+q_{2 n-2} z^{n-2}\right)+\mu, \\
& \mu r\left(\epsilon^{-1 /(n-2)} z\right)=-z^{2}\left(1+z^{n-2}\right)+\mu,
\end{aligned}
$$

and we find the $n-2$ roots with an $\mathrm{O}\left(\epsilon^{-1 /(n-2)}\right)$-modulus of $p(y)$, the $n-2$ roots with an $\mathrm{O}\left(\epsilon^{-1 /(n-2)}\right)$-modulus of $q(y)$, and the $n-2$ roots with an $\mathrm{O}\left(\epsilon^{-1 /(n-2)}\right)$-modulus of $r(y)$.

Lemma 15. If $0<\epsilon \ll 1, n \in \mathbb{N}$ with $n \geq 3$, and $y_{\star} \in \mathbb{C}$ is a root of $p(y)$ or $q(y)$ with an $\mathrm{O}\left(\epsilon^{-1 / n}\right)$-modulus, then there exists a constant $\eta_{\star} \in \mathbb{R}$ such that

$$
\left|\mathfrak{J} \xi_{\star}\right|=\frac{|\log \epsilon|}{n}+\eta_{\star}+\mathrm{O}\left(\epsilon^{2 / n} \log \epsilon\right),
$$

as $\epsilon \rightarrow 0^{+}$.

Proof. For simplicity, we assume that $y_{\star}$ is a root of $q(y)$ such that $\mathfrak{R} y_{\star} \leq 0$ and $\mathfrak{J} y_{\star} \geq 0$. Other cases require minor changes.

If $r_{0}=(n-1)^{-1 / n} / 2, r_{\star}=\epsilon^{1 / n}\left|y_{\star}\right|$, and $\theta_{\star}=\arg y_{\star}$, then $\pi / 2 \leq \theta_{\star}<n \pi /(n+1)$ and $r_{\star}=2 r_{0}+\mathrm{O}\left(\epsilon^{2 / n}\right)$, because $y_{\star}=$ $\epsilon^{-1 / n} z+\mathrm{O}\left(\epsilon^{1 / n}\right)$ for some $z \in \mathbb{C}$ such that $z^{n}=-1 /(n-1)<0$.

We compute $\xi_{\star}=\int_{0}^{y_{\star}} g(y) \mathrm{d} y$ by integrating over the path $\sigma_{\star}=\sigma_{1} \cup \sigma_{2} \cup \sigma_{3}$, where

$$
\begin{aligned}
& \sigma_{1}=\left\{\epsilon^{-1 / n} \mathrm{i} t: 0 \leq t \leq r_{0}\right\}, \\
& \sigma_{2}=\left\{\epsilon^{-1 / n} r_{0} \mathrm{e}^{\theta \mathrm{i}}: \pi / 2 \leq \theta \leq \theta_{\star}\right\}, \\
& \sigma_{3}=\left\{\epsilon^{-1 / n} \mathrm{e}^{\theta_{\star} \mathrm{i}} r: r_{0} \leq r \leq r_{\star}\right\} .
\end{aligned}
$$

This path only intersects the set of rays $\mathcal{R}$ at its endpoint $y_{\star}$, since the $2 n$ roots of $p(y)$ and $q(y)$ with an $\mathrm{O}\left(\epsilon^{-1 / n}\right)$-modulus have pairwise different arguments when $\epsilon \rightarrow 0^{+}$.

We write $\xi_{\star}=\int_{0}^{y_{\star}} g(y) \mathrm{d} y=\int_{\sigma_{\star}} g(y) \mathrm{d} y=\xi_{1}+\xi_{2}+\xi_{3}$, where

$$
\begin{aligned}
& \xi_{1}=\int_{\sigma_{1}} g(y) \mathrm{d} y=\int_{0}^{r_{0}} \epsilon^{-1 / n} \mathrm{i} g\left(\epsilon^{-1 / n} \mathrm{i} t\right) \mathrm{d} t, \\
& \xi_{2}=\int_{\sigma_{2}} g(y) \mathrm{d} y=\int_{\pi / 2}^{\theta_{\star}} \epsilon^{-1 / n} r_{0} \mathrm{e}^{\theta \mathrm{i}} \mathrm{i} g\left(\epsilon^{-1 / n} r_{0} \mathrm{e}^{\theta \mathrm{i}}\right) \mathrm{d} \theta, \\
& \xi_{3}=\int_{\sigma_{3}} g(y) \mathrm{d} y=\int_{r_{0}}^{r_{\star}} \epsilon^{-1 / n} \mathrm{e}^{\theta_{\star} \mathrm{i}} g\left(\epsilon^{-1 / n} \mathrm{e}^{\theta_{\star} \mathrm{i}} r\right) \mathrm{d} r .
\end{aligned}
$$


In order to study $\xi_{1}$, we consider the function

$$
h(t):=\epsilon^{-1 / n} \sqrt{t^{2}+\epsilon^{2 / n}} g\left(\epsilon^{-1 / n} \mathrm{i} t\right)=h_{0}(t)+\mathrm{O}\left(\epsilon^{2 / n}\right),
$$

where $h_{0}(t)=\left(1+p_{n} \mathrm{i}^{n} t^{n}\right)^{2 / 3}\left(1+q_{n} \mathrm{i}^{n} t^{n}\right)^{-1 / 2}$. The function $h_{0}(t)$ is smooth in the interval $\left[0, r_{0}\right]$ and $h_{0}(t)=1$. Besides,

$$
\xi_{1}=\mathrm{i} \int_{0}^{r_{0}}\left(t^{2}+\epsilon^{2 / n}\right)^{-1 / 2} h(t) \mathrm{d} t=\hat{\xi}_{1}+\check{\xi}_{1}+\tilde{\xi}_{1}+\breve{\xi}_{1},
$$

where

$$
\begin{aligned}
\hat{\xi}_{1} & =\mathrm{i} \int_{0}^{r_{0}} \frac{\mathrm{d} t}{\sqrt{t^{2}+\epsilon^{2 / n}}}=\mathrm{i} \arg \sinh \left(\epsilon^{-1 / n} r_{0}\right) \\
& =\mathrm{i} \frac{|\log \epsilon|}{n}+\mathrm{i} \log \left(2 r_{0}\right)+\mathrm{O}\left(\epsilon^{2 / n}\right), \\
\check{\xi}_{1} & =\mathrm{i} \int_{0}^{r_{0}} \frac{h_{0}(t)-1}{t} \mathrm{~d} t, \\
\tilde{\xi}_{1} & =\mathrm{i} \int_{0}^{r_{0}} \frac{h_{0}(t)-1}{t}\left(\frac{t}{\sqrt{t^{2}+\epsilon^{2 / n}}}-1\right) \mathrm{d} t=\mathrm{O}\left(\epsilon^{2 / n} \log \epsilon\right), \\
\breve{\xi}_{1} & =\mathrm{i} \int_{0}^{r_{0}} \frac{h(t)-h_{0}(t)}{\sqrt{t^{2}+\epsilon^{2 / n}}} \mathrm{~d} t=\mathrm{O}\left(\epsilon^{2 / n} \log \epsilon\right) .
\end{aligned}
$$

The integral $\hat{\xi}_{1}$ is immediate. The integral $\breve{\xi}_{1}$ does not depend on $\epsilon$. The integral $\tilde{\xi}_{1}$ is bounded using ideas from the proof of Lemma 23 in [45]. The integral $\breve{\xi}_{1}$ is bounded using (C.3). Hence, we have already seen that there exists $\eta_{1} \in \mathbb{R}$ such that

$$
\left|\mathfrak{J} \xi_{1}\right|=\frac{|\log \epsilon|}{n}+\eta_{1}+\mathrm{O}\left(\epsilon^{2 / n} \log \epsilon\right)
$$

The study of $\xi_{2}$ and $\xi_{3}$ is easier, because

$$
\xi_{2}=\check{\xi}_{2}+\mathrm{O}\left(\epsilon^{2 / n}\right), \quad \xi_{3}=\check{\xi}_{3}+\mathrm{O}\left(\epsilon^{2 / n}\right),
$$

for some constants $\breve{\xi}_{2}$ and $\breve{\xi}_{3}$ that do not depend on $\epsilon$.

For instance, $\xi_{2}$ depends on $\epsilon^{2 / n}$ in an analytic way, because the integrand $\epsilon^{-1 / n} r_{0} \mathrm{e}^{\theta \mathrm{i}} \mathrm{i} g\left(\epsilon^{-1 / n} r_{0} \mathrm{e}^{\theta \mathrm{i}}\right)$ and the argument $\theta_{\star}$ are both analytic in $\epsilon^{2 / n}$, and all the singularities of the integrand are far from the integration path. The study of $\xi_{3}$ is similar.

Finally, if $\delta$ is the distance of the set of singularities and zeros of the curvature $\kappa(\xi)$ to the real axis, then

$$
\begin{aligned}
2 \pi \delta & =\frac{2 \pi}{C} \min \left\{\left|\Im \xi_{\star}\right|: y_{\star} \text { is a root with an } \mathrm{O}\left(\epsilon^{-1 / n}\right) \text {-modulus }\right\} \\
& =\frac{|\log \epsilon|}{n}+\eta+\mathrm{O}\left(\epsilon^{2 / n} \log \epsilon\right)
\end{aligned}
$$

where the constant $\eta=\eta_{n} \in \mathbb{R}$ is equal to the smallest constant $\eta_{\star}$ provided by Lemma 15 among all the roots of $p(y)$ and $q(y)$ with an $\mathrm{O}\left(\epsilon^{-1 / n}\right)$-modulus. We have also used relation (C.2) in the last equality.

We do not care about the roots $y_{ \pm}= \pm 1+\mathrm{O}(\epsilon)$ of $r(y)$, since they correspond to points where $y$ is not a true coordinate over the perturbed circle $Q$. To be precise, the points $\left(0, y_{ \pm}\right)$are the two vertices of $Q$ over the symmetry line $\{x=0\}$, and the curvature has a finite positive value at them. Nor do we care about the roots whose modulus is $\mathrm{O}\left(\epsilon^{-1 /(n-2)}\right)$, because

$$
\epsilon^{-1 /(n-2)} g\left(\epsilon^{-1 /(n-2)} z\right)=\epsilon^{-1 /(3 n-6)}\left(l_{0}(z)+\mathrm{o}(1)\right),
$$

where

$$
l_{0}(z)=\frac{z^{n / 6-1}\left(p_{n}+p_{2 n-2} z^{n-2}\right)^{2 / 3}}{\left(1+z^{n-2}\right)^{1 / 2}\left(q_{n}+q_{2 n-2} z^{n-2}\right)^{1 / 2}} .
$$

This implies that, if $y_{\star} \in \mathbb{C}$ is one of those farther roots, then

$$
\left|\mathfrak{J} \xi_{\star}\right|=\epsilon^{-1 /(3 n-6)}\left(v_{\star}+\mathrm{O}(1)\right)
$$

for some constant $v_{\star} \in \mathbb{R}$. That is, the farther roots give rise to much bigger imaginary parts.

\section{References}

[1] G. D. Birkhoff, Dynamical Systems. With an addendum by Jurgen Moser. American Mathematical Society Colloquium Publications, Vol. IX, Providence, R.I.: American Mathematical Society, 1966.

[2] A. Katok and B. Hasselblatt, Introduction to the Modern Theory of Dynamical Systems, vol. 54 of Encyclopedia of Mathematics and its Applications. Cambridge: Cambridge University Press, 1995.

[3] V. Kozlov and D. Treschev, Billiards: A Genetic Introduction to the Dynamics of Systems with Impacts, vol. 89 of Translations of Mathematical Monographs. Providence, RI: Amer. Math. Soc., 1991.

[4] S. Tabachnikov, "Billiards," Panor. Synth., no. 1, pp. vi+142, 1995.

[5] K. G. Andersson and R. B. Melrose, "The propagation of singularities along gliding rays," Invent. Math., vol. 41, no. 3, pp. 197-232, 1977.

[6] M. Kac, "Can one hear the shape of a drum?," Amer. Math. Monthly, vol. 73, no. 4, part II, pp. 1-23, 1966.

[7] K. F. Siburg, The principle of least action in geometry and dynamics, vol. 1844 of Lecture Notes in Mathematics. Berlin: Springer-Verlag, 2004.

[8] R. S. MacKay, J. D. Meiss, and I. C. Percival, "Transport in Hamiltonian systems," Phys. D, vol. 13, no. 1-2, pp. 55-81, 1984.

[9] J. N. Mather, "A criterion for the nonexistence of invariant circles," Inst. Hautes Études Sci. Publ. Math., no. 63, pp. 153-204, 1986.

[10] J. D. Meiss, "Symplectic maps, variational principles, and transport," Rev. Modern Phys., vol. 64, no. 3, pp. 795-848, 1992.

[11] J. N. Mather and G. Forni, "Action minimizing orbits in Hamiltonian systems," in Transition to chaos in classical and quantum mechanics (Montecatini Terme, 1991), vol. 1589 of Lecture Notes in Math., pp. 92-186, Springer, Berlin, 1994.

[12] S. Marvizi and R. Melrose, "Spectral invariants of convex planar regions," J. Differential Geom., vol. 17, no. 3, pp. 475-502, 1982.

[13] A. Sorrentino, "Computing Mather's $\beta$-function for Birkhoff billiards," Discrete Contin. Dyn. Syst., vol. 35, no. 10, pp. 5055-5082, 2015.

[14] P. Martín, R. Ramírez-Ros, and A. Tamarit-Sariol, "On the length and area spectrum of analytic convex domains," ArXiv e-prints, Oct. 2014.

[15] J. M. Greene, "A method for determining a stochastic transition," J. Math. Phys., vol. 20, pp. 1183-1201, 1979.

[16] R. S. MacKay, "Greene's residue criterion," Nonlinearity, vol. 5, no. 1, pp. 161-187, 1992.

[17] A. Delshams and R. de la Llave, "KAM theory and a partial justification of Greene's criterion for nontwist maps," SIAM J. Math. Anal., vol. 31, no. 6, pp. 1235-1269, 2000.

[18] E. Fontich and C. Simó, "The splitting of separatrices for analytic diffeomorphisms," Ergodic Theory Dynam. Systems, vol. 10, no. 2, pp. 295318, 1990.

[19] V. G. Gelfreich, V. F. Lazutkin, and M. B. Tabanov, "Exponentially small splittings in Hamiltonian systems," Chaos, vol. 1, no. 2, pp. 137-142, 1991.

[20] A. Delshams and R. Ramírez-Ros, "Exponentially small splitting of separatrices for perturbed integrable standard-like maps," Journal of Nonlinear Science, vol. 8, no. 3, pp. 317-352, 1998.

[21] A. Delshams and R. Ramírez-Ros, "Singular separatrix splitting and the Melnikov method: an experimental study," Experimental Mathematics, vol. 8, no. 1, pp. 29-48, 1999.

[22] V. G. Gelfreich, "A proof of the exponentially small transversality of the separatrices for the standard map," Communications in Mathematical Physics, vol. 201, no. 1, pp. 155-216, 1999. 
[23] V. G. Gelfreich and V. F. Lazutkin, "Splitting of separatrices: perturbation theory and exponential smallness," Russian Mathematical Surveys, vol. 56, no. 3, pp. 499-558, 2001.

[24] V. Gelfreich and D. Sauzin, "Borel summation and splitting of separatrices for the Hénon map," Ann. Inst. Fourier (Grenoble), vol. 51, no. 2, pp. 513-567, 2001.

[25] R. Ramírez-Ros, "Exponentially small separatrix splittings and almost invisible homoclinic bifurcations in some billiard tables," Phys. D, vol. 210, no. 3-4, pp. 149-179, 2005.

[26] V. Gelfreich and C. Simó, "High-precision computations of divergent asymptotic series and homoclinic phenomena," Discrete Contin. Dyn. Syst. Ser. B, vol. 10, no. 2-3, pp. 511-536, 2008.

[27] P. Martín, D. Sauzin, and T. M. Seara, "Exponentially small splitting of separatrices in the perturbed McMillan map," Discrete Contin. Dyn. Syst., vol. 31, no. 2, pp. 301-372, 2011

[28] P. Martín, D. Sauzin, and T. M. Seara, "Resurgence of inner solutions for perturbations of the McMillan map," Discrete Contin. Dyn. Syst., vol. 31, no. 1, pp. 165-207, 2011.

[29] I. Baldomá and P. Martín, "The inner equation for generalized standard maps,” SIAM J. Appl. Dyn. Syst., vol. 11, no. 3, pp. 1062-1097, 2012.

[30] H. Poritsky, "The billiard ball problem on a table with a convex boundary—an illustrative dynamical problem," Ann. of Math. (2), vol. 51, pp. 446-470, 1950.

[31] A. Delshams and R. Ramírez-Ros, "Poincaré-Melnikov-Arnold method for analytic planar maps," Nonlinearity, vol. 9, no. 1, pp. 1-26, 1996.

[32] A. Avila, J. D. Simoi, and V. Kaloshin, "An integrable deformation of an ellipse of small eccentricity is an ellipse," ArXiv e-prints, Dec. 2014.

[33] R. Ramírez-Ros, "Break-up of resonant invariant curves in billiards and dual billiards associated to perturbed circular tables," Phys. D, vol. 214, no. 1 , pp. 78-87, 2006.

[34] S. Pinto-de-Carvalho and R. Ramírez-Ros, "Non-persistence of resonant caustics in perturbed elliptic billiards," Ergodic Theory Dynam. Systems, vol. 33, no. 6, pp. 1876-1890, 2013.

[35] C. Batut, K. Belabas, D. Bernardi, H. Cohen, and M. Olivier, "User's Guide to PARI/G," 2006.

[36] V. F. Lazutkin, "Existence of caustics for the billiard problem in a convex domain," Izv. Akad. Nauk SSSR Ser. Mat., vol. 37, pp. 186-216, 1973.

[37] A. Olvera, "Estimation of the amplitude of resonance in the general standard map," Experiment. Math., vol. 10, no. 3, pp. 401-418, 2001.

[38] M. Guardia and T. M. Seara, "Exponentially and non-exponentially small splitting of separatrices for the pendulum with a fast meromorphic perturbation," Nonlinearity, vol. 25, no. 5, p. 1367, 2012

[39] A. Delshams and T. M. Seara, "An asymptotic expression for the splitting of separatrices of the rapidly forced pendulum," Comm. Math. Phys., vol. 150 , no. 3, pp. 433-463, 1992.

[40] A. Delshams and T. M. Seara, "Splitting of separatrices in Hamiltonian systems with one and a half degrees of freedom," Math. Phys. Electron. J., vol. 3, pp. Paper 4, 40, 1997.

[41] M. Guardia, C. Olivé, and T. M. Seara, "Exponentially small splitting for the pendulum: a classical problem revisited," J. Nonlinear Sci., vol. 20, no. 5, pp. 595-685, 2010.

[42] M. Abramowitz and I. A. Stegun, Handbook of Mathematical Functions with Formulas, Graphs, and Mathematical Tables, vol. 55 of National Bureau of Standards Applied Mathematics Series. U.S. Government Printing Office, Washington, D.C., 1964.

[43] E. T. Whittaker and G. N. Watson, A Course of Modern Analysis. Cambridge Mathematical Library, Cambridge: Cambridge University Press, 1996. An introduction to the general theory of infinite processes and of analytic functions; with an account of the principal transcendental functions, Reprint of the fourth (1927) edition.

[44] S. Chang and R. Friedberg, "Elliptical billiards and Poncelet's theorem," J. Math. Phys., vol. 29, no. 7, pp. 1537-1550, 1988.

[45] P. S. Casas and R. Ramírez-Ros, "The frequency map for billiards inside ellipsoids," SIAM J. Appl. Dyn. Syst., vol. 10, no. 1, pp. 278-324, 2011. 